\title{
Three Reversible Redox States of Thiolate-Bridged Dirhodium Complexes without Metal-Metal Bonds
}

Ryan P. Coll, Kim R. Dunbar

\section{Supporting Information}

\section{Contents}

Crystallographic refinement details

$\mathrm{X}$-ray crystal structures with $\mathrm{Rh}, \mathrm{S}$, and $\mathrm{N}$ atom labels.

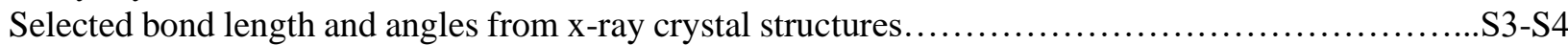

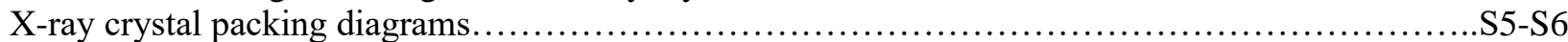

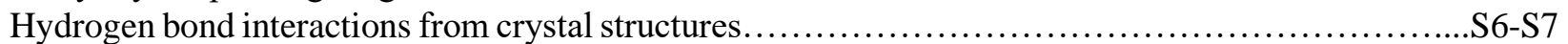

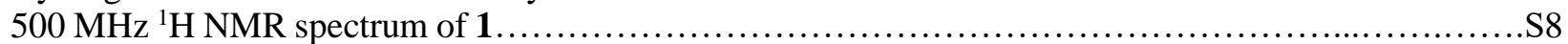

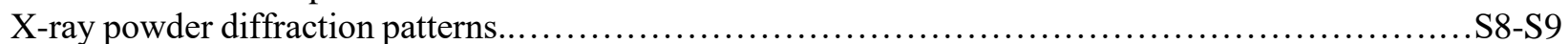

SHAPE-calculated continuous shape measurement tables...........................................

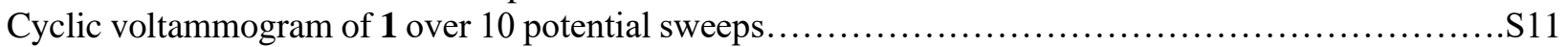

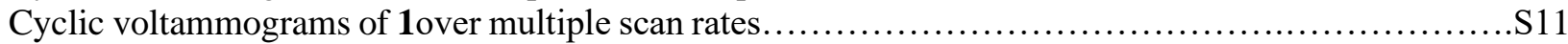

Electrochemical data from cyclic voltammograms of $\mathbf{1}$ over multiple scan rates.......................S12

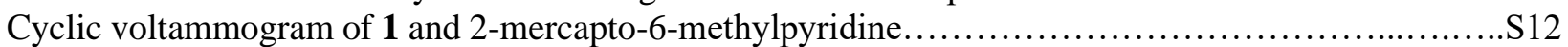

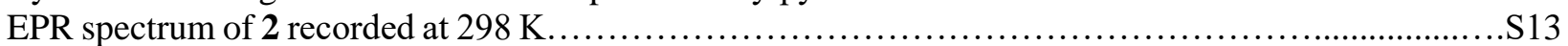

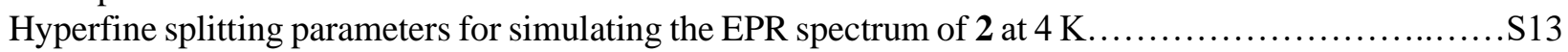

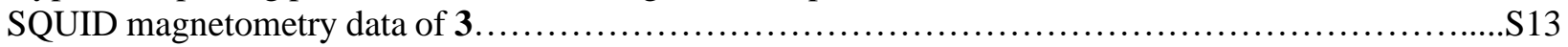

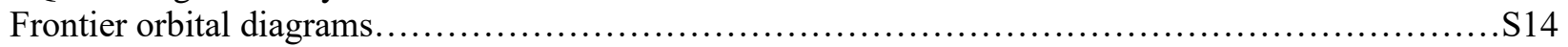

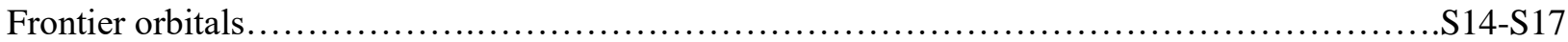

Cartesian coordinates of gas phase-optimized structures................................S17-S22

Structural comparisons between crystal and optimized structures............................S23-S25

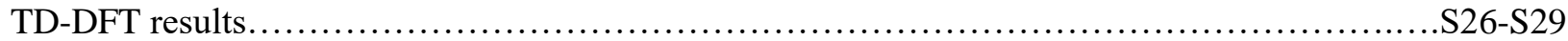

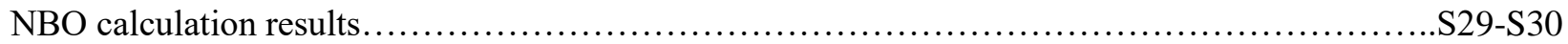

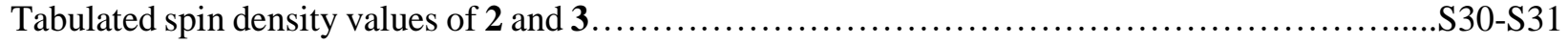

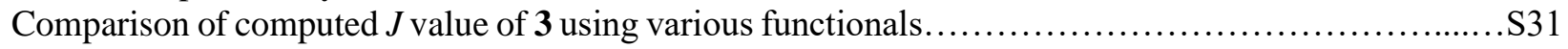

Magneto-structure correlation of $\mathbf{3}$ with Mulliken point charges (effect of structural manipulation on

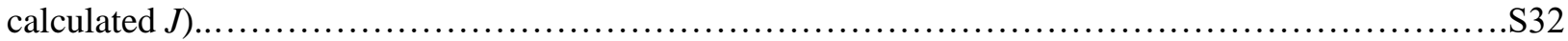

Magneto-structure correlation of $\mathbf{3}$ with Mulliken point charges (effect of structural manipulation on singlettriplet energy gap).... 
Table S1. Crystallographic refinement details for 1, 2, and 3.

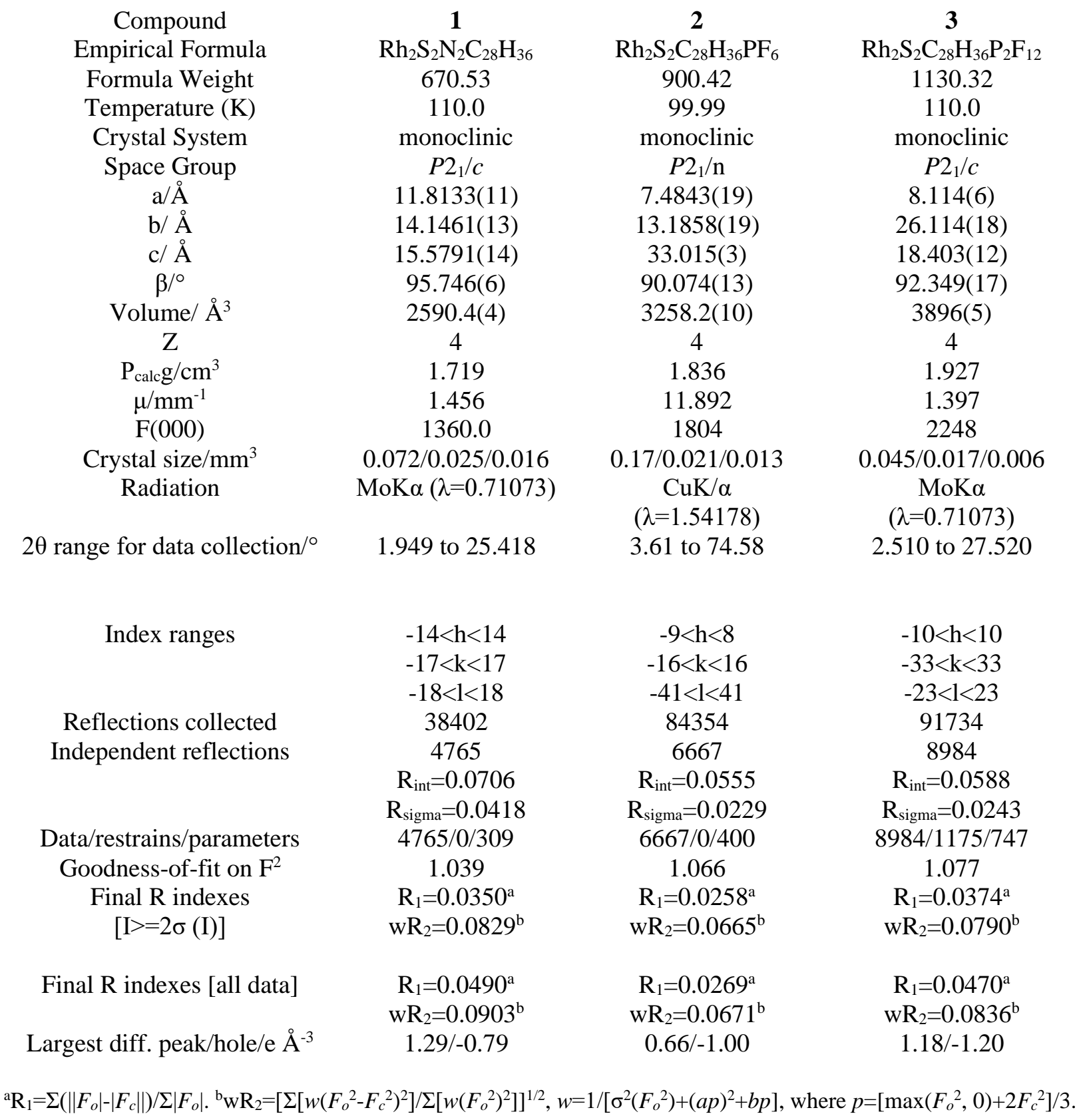




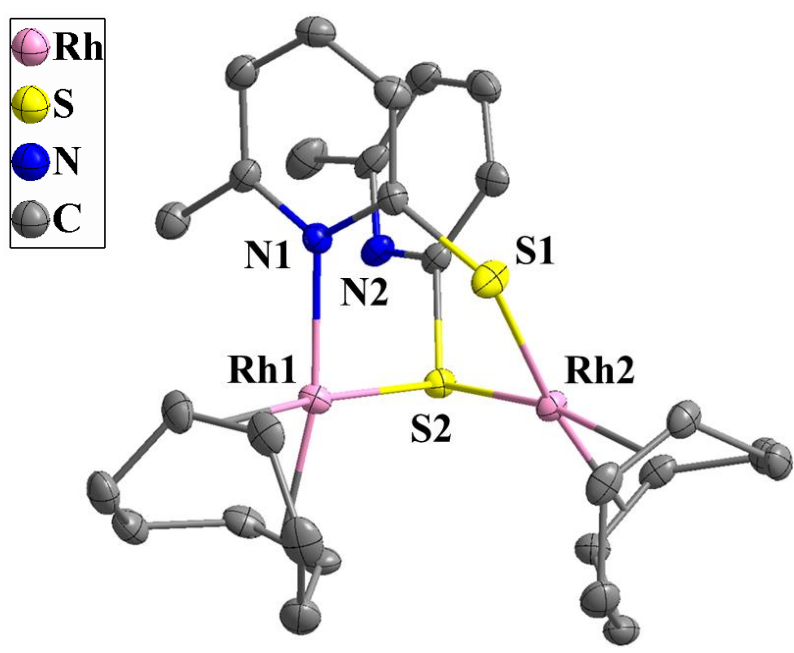

Figure S1. The X-ray crystal structure of $\mathbf{1}$. Atoms are represented by anisotropically-refined thermal ellipsoids at 50\% probability. Hydrogen atoms are omitted for clarity.

Table S2. Selected bond lengths and angles from the crystal structure of $\mathbf{1 .}$

$\begin{array}{cccc}\text { Bond } & \AA & \text { Angle } & \circ \\ \text { Rh1-N1 } & 2.119(3) & \text { N1-Rh1-S2 } & 90.23(9) \\ \text { Rh1-S2 } & 2.3542(11) & \text { Rh1-S2-Rh2 } & 81.98(4) \\ \text { Rh2-S1 } & 2.3499(11) & \text { S1-Rh2-S2 } & 100.95(4) \\ \text { Rh2-S2 } & 2.3726(11) & & \end{array}$

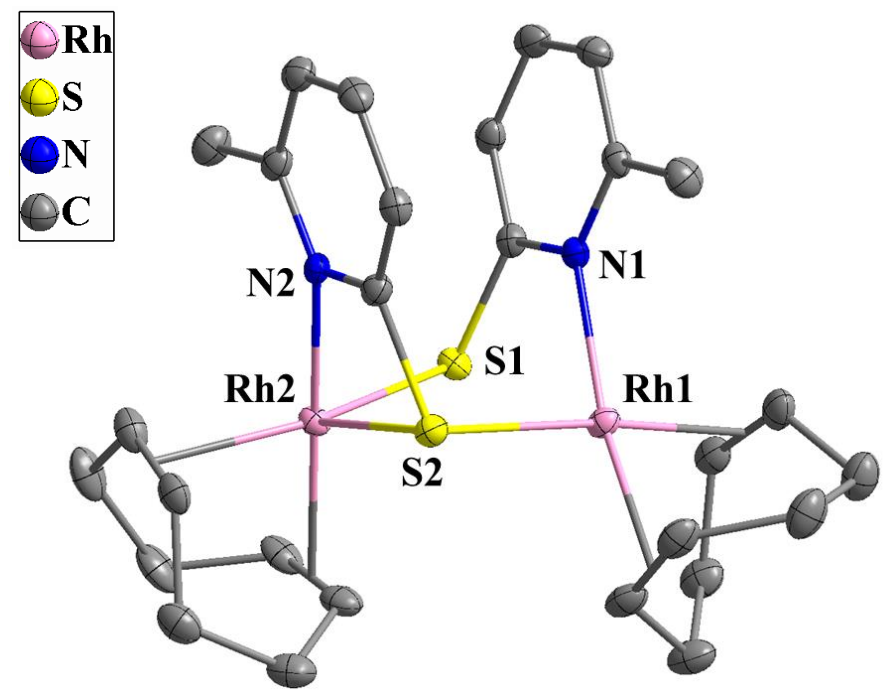

Figure S2. The X-ray crystal structure of $\mathbf{2}$. Atoms are represented by anisotropically-refined thermal ellipsoids at 50\% probability. Hydrogen atoms are omitted for clarity. 
Table S3. Selected bond lengths and angles from the crystal structure of 2.

$\begin{array}{cccc}\text { Bond } & \AA & \text { Angle } & \circ \\ \text { Rh1-N1 } & 2.123(3) & \text { N1-Rh1-S2 } & 93.27(8) \\ \text { Rh1-S2 } & 2.3596(9) & \text { Rh1-S2-Rh2 } & 91.98(3) \\ \text { Rh2-N2 } & 2.079(3) & \text { N2-Rh2-S1 } & 93.14(8) \\ \text { Rh2-S1 } & 2.3374(9) & \text { N2-Rh2-S2 } & 66.67(9) \\ \text { Rh2-S2 } & 2.5677(11) & & \end{array}$

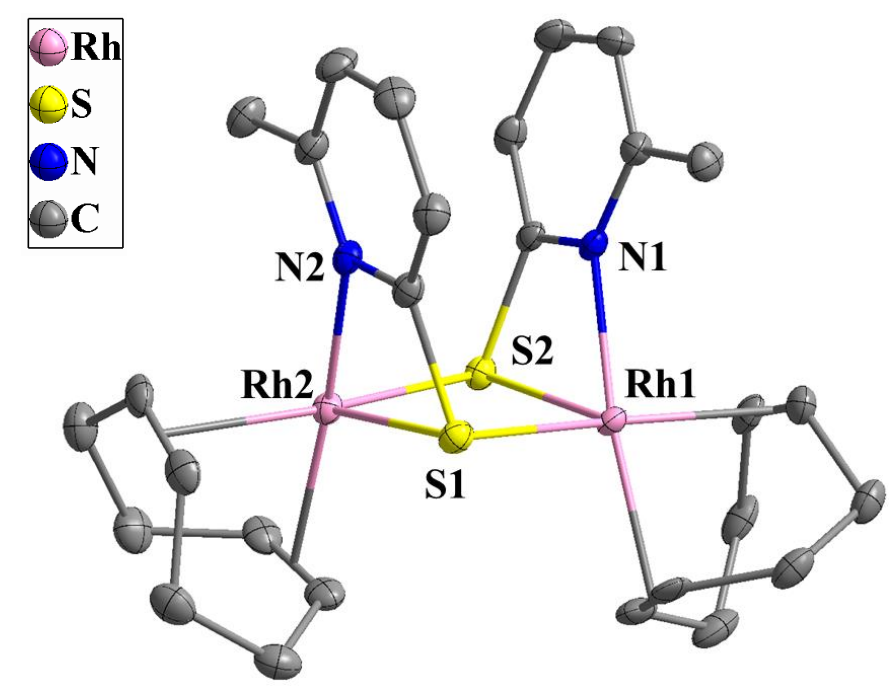

Figure S3. The X-ray crystal structure of $\mathbf{3}$. Atoms are represented by anisotropically-refined thermal ellipsoids at $50 \%$ probability. Hydrogen atoms are omitted for clarity.

Table S4. Selected bond lengths and angles from the crystal structure of $\mathbf{3}$.

$\begin{array}{cccc}\text { Bond } & \AA & \text { Angle } & \circ \\ \text { Rh1-N1 } & 2.075(3) & \text { N1-Rh1-S1 } & 95.27(8) \\ \text { Rh1-S1 } & 2.3107(16) & \text { N1-Rh1-S2 } & 67.01(8) \\ \text { Rh1-S2 } & 2.5197(16) & \text { Rh1-S1-Rh2 } & 86.80(5) \\ \text { Rh2-N2 } & 2.074(3) & \text { Rh1-S2-Rh2 } & 86.92(5) \\ \text { Rh2-S1 } & 2.5381(16) & \text { S1-Rh1-S2 } & 93.27(5) \\ \text { Rh2-S2 } & 2.3252(16) & \text { S1-Rh2-S2 } & 92.45(5) \\ & & \text { N2-Rh2-S1 } & 66.89(8) \\ & & \text { N2-Rh2-S2 } & 95.15(8)\end{array}$




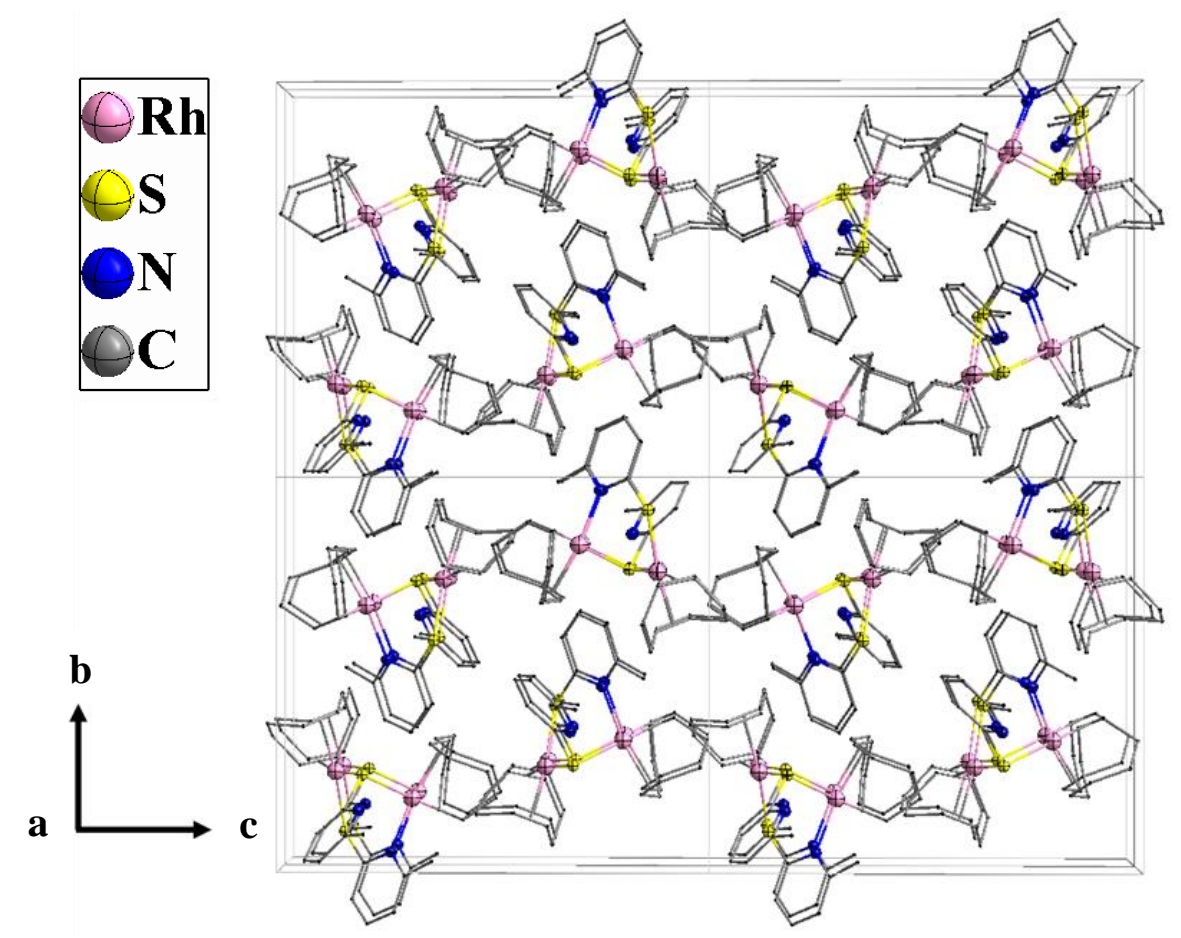

Figure S4. X-ray crystal packing for 1 along the $a$ axis.

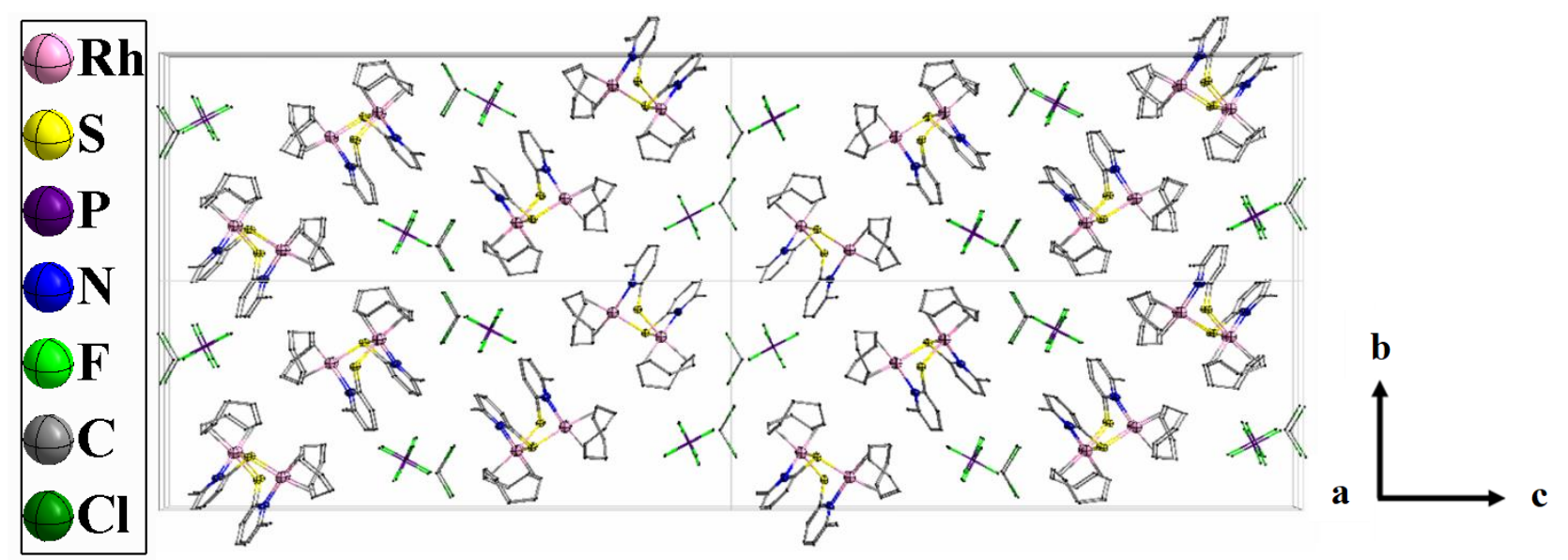

Figure S5. X-ray crystal packing for $\mathbf{2}$ along the $a$ axis. 


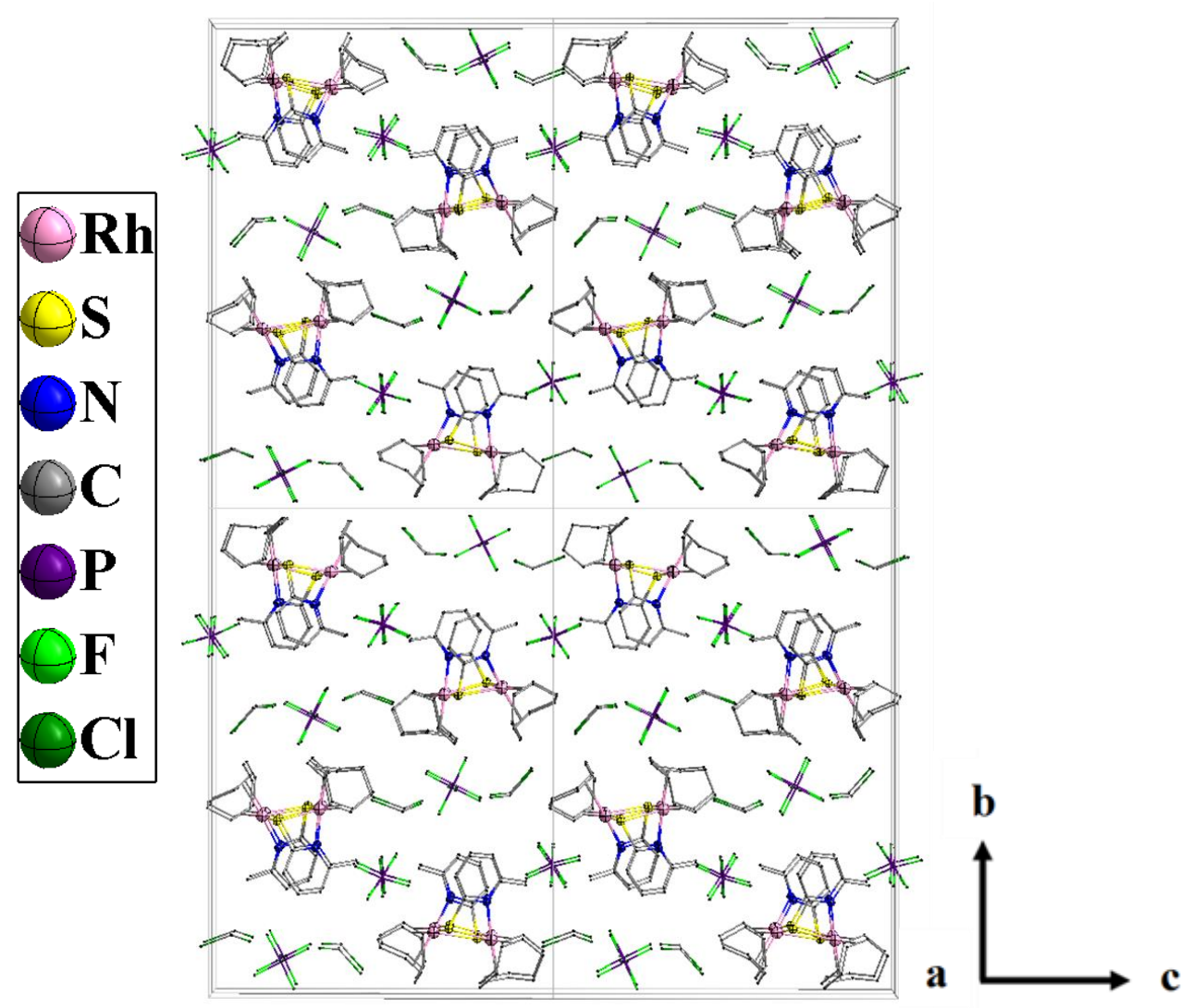

Figure S6. X-ray crystal packing for $\mathbf{3}$ along the $a$ axis.

Table S5. Hydrogen bond interactions in the crystal structure of 2 ( $\mathrm{D}=$ donor atom, H=hydrogen, $\mathrm{A}=$ acceptor atom).

$\begin{array}{cccccc}\text { D-H } & \text { D-H }(\AA) & \text { H-A }(\AA) & \text { D-H-A Angle }\left(^{\circ}\right) & \text { D-A }(\AA) & \text { A } \\ \text { C5-H5 } & 0.950 & 2.835 & 162.94 & 3.753 & \text { S1 [x-1,y, z] } \\ \text { C3-H3 } & 0.950 & 2.974 & 162.15 & 3.889 & \text { C12 [-x+1, -y+2, -z] } \\ \text { C11-H11 } & 0.950 & 2.636 & 123.52 & 3.258 & \text { F6 [x+1,y, z] } \\ \text { C9-H9 } & 0.950 & 2.558 & 134.30 & 3.293 & \text { F6 } \\ \text { C7-H7B } & 0.980 & 2.407 & 156.04 & 3.326 & \text { F6 } \\ \text { C29-H29A } & 0.990 & 2.441 & 145.60 & 3.305 & \text { F2 [-x, -y+2, -z] } \\ \text { C29-H29B } & 0.990 & 2.509 & 119.33 & 3.116 & \text { F4 [-x+1,-y+2,-z]] }\end{array}$


Table S6. Hydrogen bond interactions in the crystal structure of $\mathbf{3}$ ( $\mathrm{D}=$ donor atom, H=hydrogen, $\mathrm{A}=$ acceptor atom).

\begin{tabular}{|c|c|c|c|c|c|}
\hline D-H & D-H $(\AA)$ & H-A $(\AA)$ & D-H-A Angle ( $\left.{ }^{\circ}\right)$ & D-A $(\AA)$ & $\mathbf{A}$ \\
\hline C10-H10 & 0.930 & 2.428 & 131.89 & 3.126 & $\mathrm{~F} 4 \mathrm{~d}[\mathrm{x}+1,-\mathrm{y}+3 / 2, \mathrm{z}+1 / 2]$ \\
\hline C10-H10 & 0.930 & 2.585 & 151.87 & 3.433 & F5A_e $[\mathrm{x}+1,-\mathrm{y}+3 / 2, \mathrm{z}+1 / 2]$ \\
\hline C10-H10 & 0.930 & 2.546 & 125.50 & 3.178 & $\mathrm{~F} 4 \mathrm{~B} \_\mathrm{f}[\mathrm{x}+1,-\mathrm{y}+3 / 2, \mathrm{z}+1 / 2]$ \\
\hline C4-H4 & 0.930 & 2.380 & 158.55 & 3.264 & F1_d \\
\hline $\mathrm{C} 4-\mathrm{H} 4$ & 0.930 & 2.518 & 169.73 & 3.438 & F1A_e \\
\hline C4-H4 & 0.930 & 2.339 & 151.64 & 3.188 & F1B_f \\
\hline $\mathrm{C} 8-\mathrm{H} 8 \mathrm{~A}$ & 0.960 & 2.629 & 163.21 & 3.559 & F5_d $[x,-y+3 / 2, z+1 / 2]$ \\
\hline $\mathrm{C} 8-\mathrm{H} 8 \mathrm{~A}$ & 0.960 & 2.467 & 155.20 & 3.363 & F6A_e $[x,-y+3 / 2, z+1 / 2]$ \\
\hline $\mathrm{C} 8-\mathrm{H} 8 \mathrm{~A}$ & 0.960 & 2.462 & 136.62 & 3.227 & F6B_f $[x,-y+3 / 2, z+1 / 2]$ \\
\hline $\mathrm{C} 8-\mathrm{H} 8 \mathrm{C}$ & 0.960 & 2.485 & 151.65 & 3.361 & F3A_e $[\mathrm{x}+1,-\mathrm{y}+3 / 2, \mathrm{z}+1 / 2]$ \\
\hline $\mathrm{C} 8-\mathrm{H} 8 \mathrm{C}$ & 0.960 & 2.471 & 130.06 & 3.175 & $\mathrm{~F} 3 \mathrm{~B} \_\mathrm{f}[\mathrm{x}+1,-\mathrm{y}+3 / 2, \mathrm{z}+1 / 2]$ \\
\hline C14-H14A & 0.960 & 2.582 & 165.74 & 3.521 & F6_d $[\mathrm{x}+1, \mathrm{y}, \mathrm{z}]$ \\
\hline C14-H14A & 0.960 & 2.570 & 165.10 & 3.507 & $\mathrm{~F} 3 \mathrm{~B} \_\mathrm{f}[\mathrm{x}+1, \mathrm{y}, \mathrm{z}]$ \\
\hline C14-H14B & 0.960 & 2.294 & 140.47 & 3.095 & F6A_e \\
\hline C14-H14C & 0.960 & 2.334 & 159.06 & 3.249 & F8_a $[-x+1, y-1 / 2,-z-1 / 2]$ \\
\hline $\mathrm{C} 12-\mathrm{H} 12$ & 0.930 & 2.360 & 146.57 & 3.178 & F9A_b $[-\mathrm{x}+1, \mathrm{y}-1 / 2,-\mathrm{z}-1 / 2]$ \\
\hline $\mathrm{C} 12-\mathrm{H} 12$ & 0.930 & 2.267 & 134.93 & 2.997 & F8B_c $[-x+1, y-1 / 2,-z-1 / 2]$ \\
\hline C32-H32A & 0.970 & 2.551 & 141.81 & 3.367 & F2_d $[-\mathrm{x},-\mathrm{y}+2,-\mathrm{z}-1]$ \\
\hline $\mathrm{C} 32-\mathrm{H} 32 \mathrm{~A}$ & 0.970 & 2.560 & 141.79 & 3.376 & F2A_e $[-x,-y+2,-z-1]$ \\
\hline C32-H32A & 0.970 & 2.290 & 162.01 & 3.227 & F4A_e $[-x,-y+2,-z-1]$ \\
\hline $\mathrm{C} 32-\mathrm{H} 32 \mathrm{~A}$ & 0.970 & 1.969 & 147.19 & 2.834 & $\mathrm{~F} 2 \mathrm{~B} \_\mathrm{f}[-\mathrm{x},-\mathrm{y}+2,-\mathrm{z}-1]$ \\
\hline C32-H32B & 0.970 & 2.484 & 147.21 & 3.341 & F12_a $[\mathrm{x}-1, \mathrm{y}, \mathrm{z}]$ \\
\hline C32-H32B & 0.970 & 2.251 & 162.51 & 3.189 & F10A_b $[\mathrm{x}-1, \mathrm{y}, \mathrm{z}]$ \\
\hline $\mathrm{C} 32-\mathrm{H} 32 \mathrm{~B}$ & 0.970 & 2.413 & 160.61 & 3.343 & F10B_c $[\mathrm{x}-1, \mathrm{y}, \mathrm{z}]$ \\
\hline C31-H31A & 0.970 & 2.336 & 139.72 & 3.139 & F9_a $[-\mathrm{x}+1,-\mathrm{y}+2,-\mathrm{z}]$ \\
\hline $\mathrm{C} 31-\mathrm{H} 31 \mathrm{~A}$ & 0.970 & 2.450 & 138.74 & 3.243 & F9A_b $[-\mathrm{x}+1,-\mathrm{y}+2,-\mathrm{z}]$ \\
\hline C31-H31A & 0.970 & 2.601 & 139.57 & 3.398 & F8B_c $[-\mathrm{x}+1,-\mathrm{y}+2,-\mathrm{z}]$ \\
\hline C31-H31B & 0.970 & 2.211 & 136.29 & 2.988 & F6_d $[x+1,-y+3 / 2, z+1 / 2]$ \\
\hline
\end{tabular}




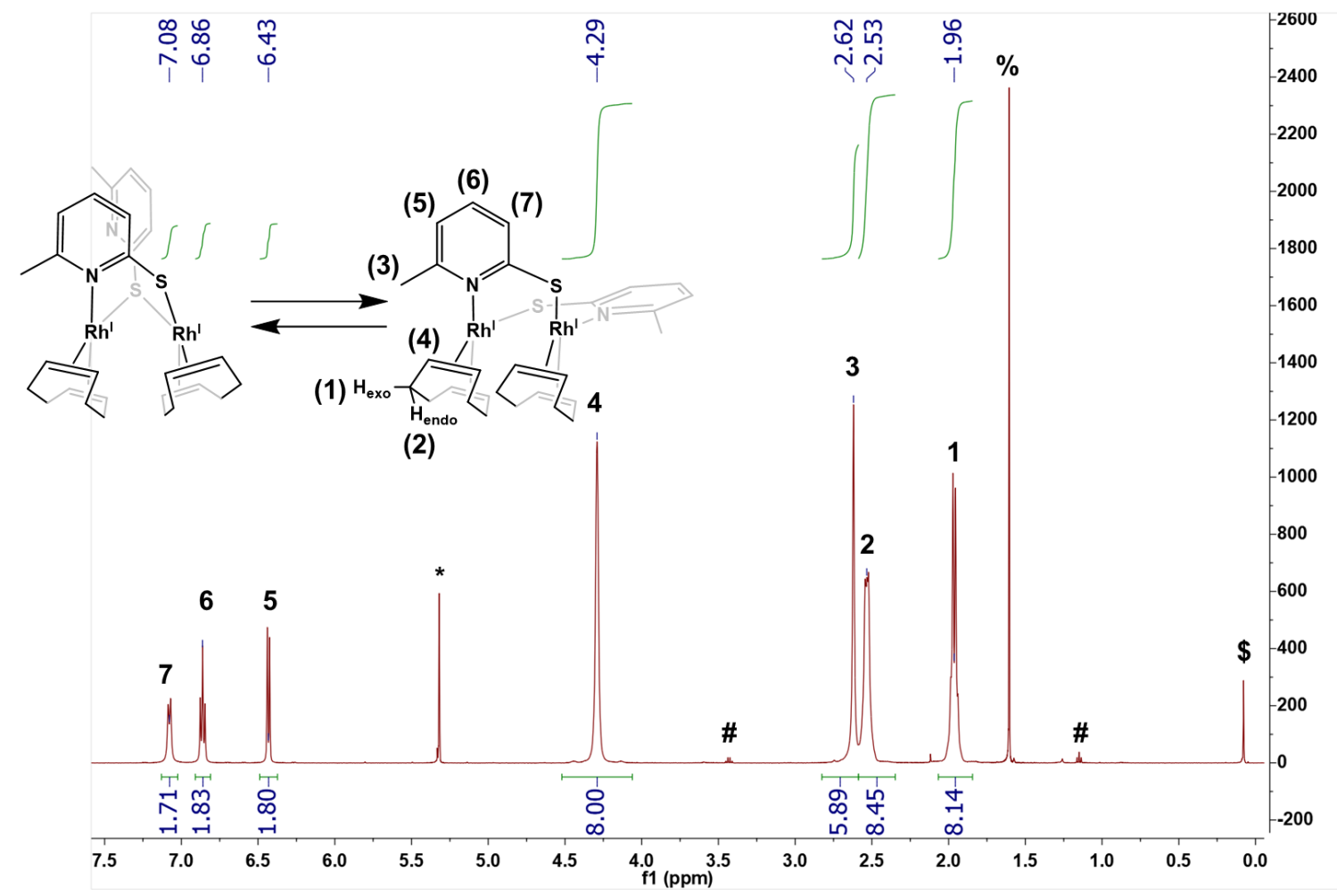

Figure S7. ${ }^{1} \mathrm{H}$ NMR spectrum of $\mathbf{1}$ (depicted as interconverting bridging arrangements as would be present in solution $)$ in dichloromethane- $d_{2}(500 \mathrm{MHz})\left({ }^{*}=\mathrm{CD}_{2} \mathrm{Cl}_{2}\right.$ residual peak, \#=diethyl ether, $\%=\mathrm{H}_{2} \mathrm{O}$, $\$=$ tetramethylsilane).

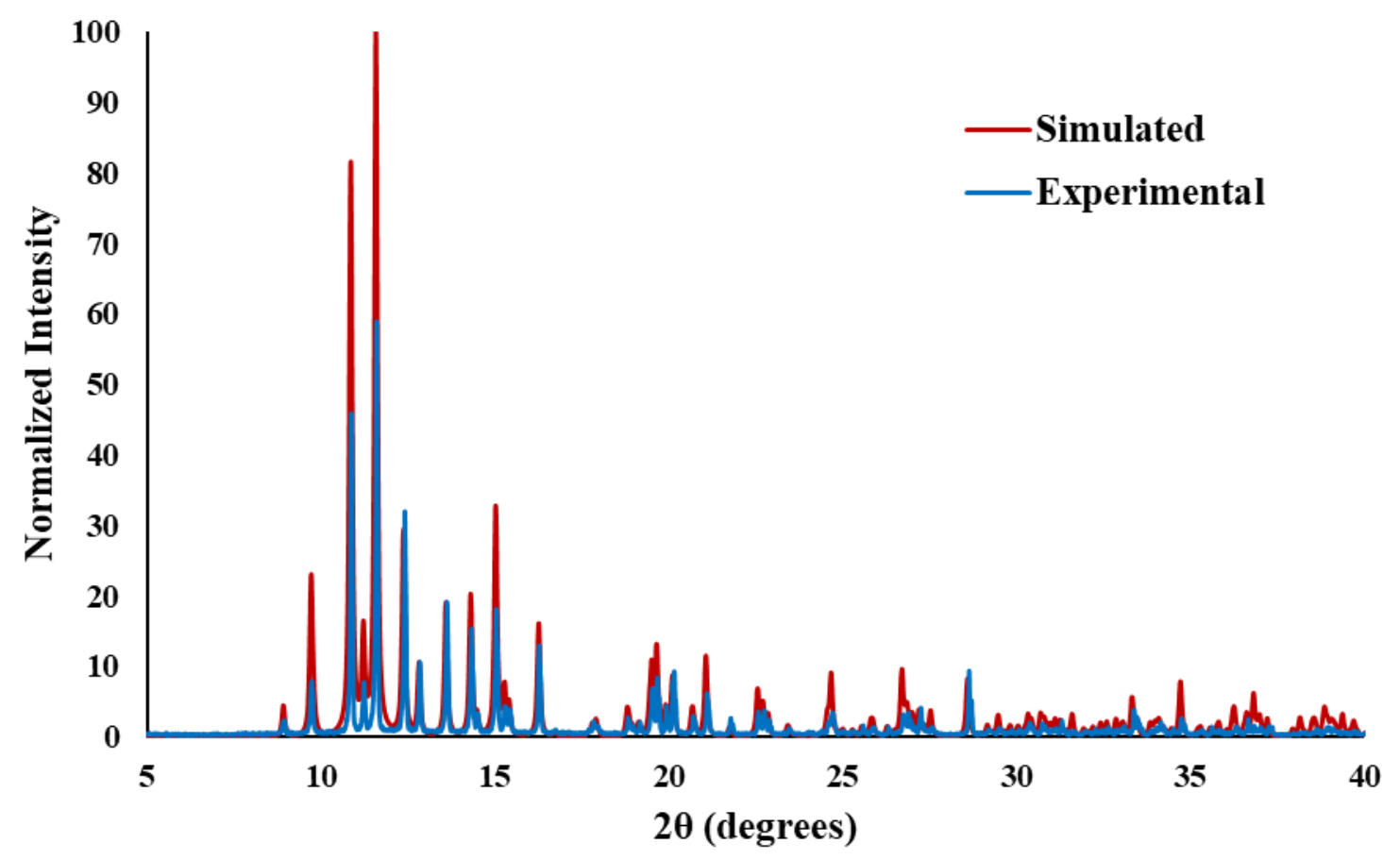

Figure S8. X-ray powder diffraction of $\mathbf{1}$. 


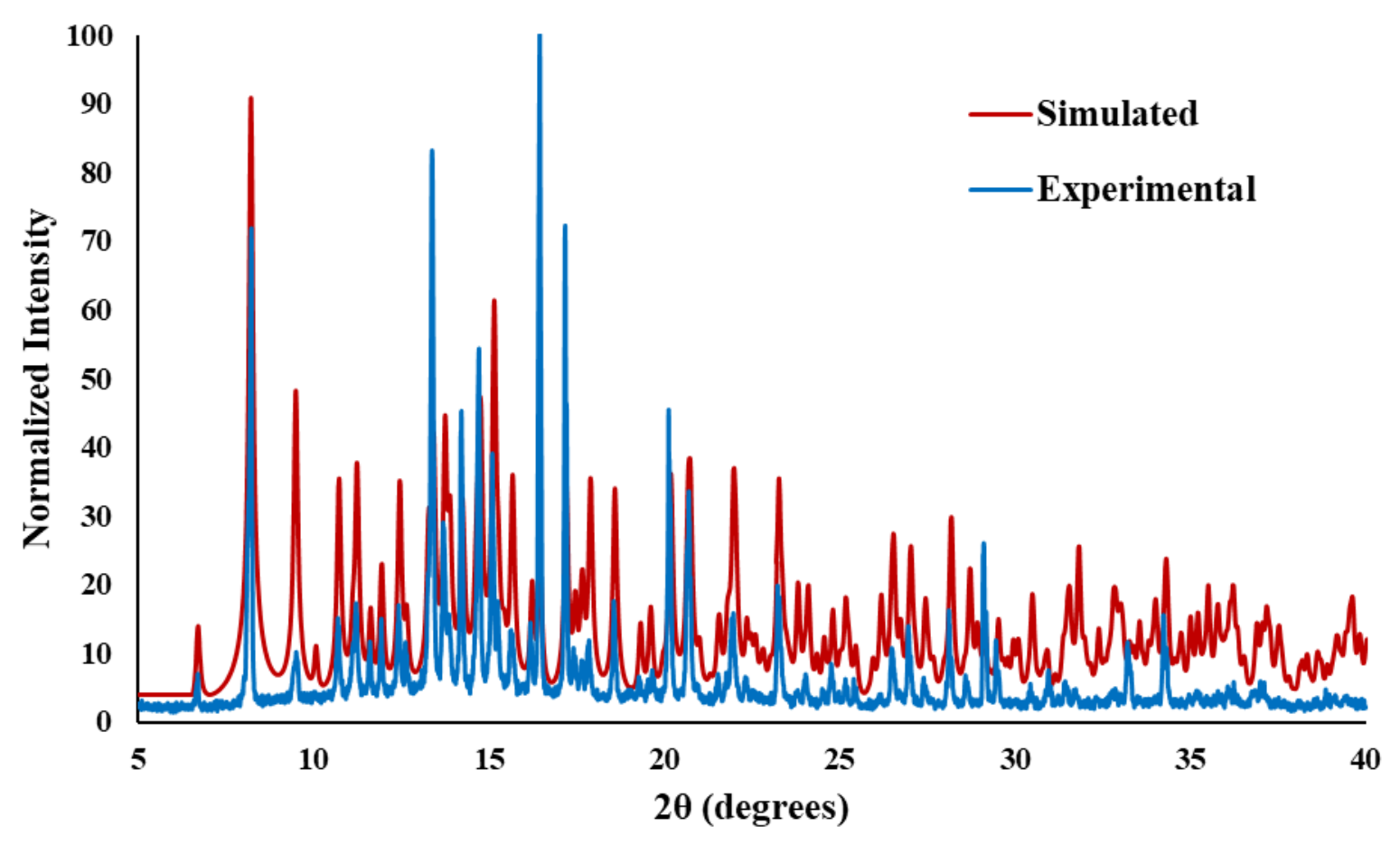

Figure S9. X-ray powder diffraction of 2.

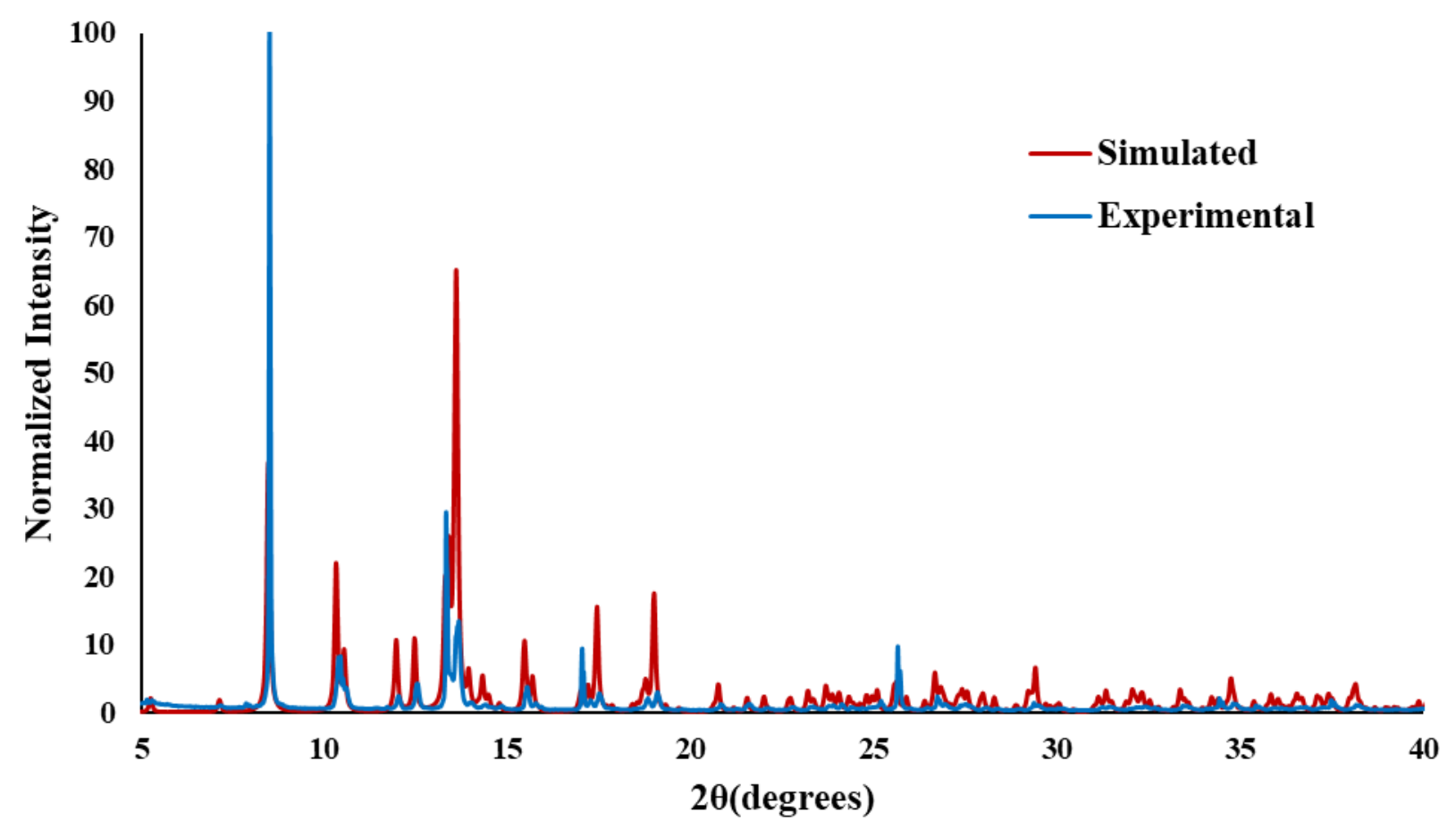

Figure S10. X-ray powder diffraction of $\mathbf{3}$. 
Table S7. SHAPE-calculated continuous shape measurements for $\mathrm{Rh} 1$ and $\mathrm{Rh} 2$ in $\mathbf{1}$ (the underlined value indicates the closest match to an idealized coordination geometry).

\begin{tabular}{|c|c|c|c|c|}
\hline $\begin{array}{c}\text { Rh1 } \\
\text { (4-coordinate) }\end{array}$ & $\begin{array}{c}\text { Square Planar } \\
\left(\boldsymbol{D}_{4 h}\right)\end{array}$ & $\begin{array}{c}\text { Tetrahedron } \\
\left(\boldsymbol{T}_{\boldsymbol{d}}\right)\end{array}$ & $\begin{array}{c}\text { Seesaw } \\
\left(\boldsymbol{C}_{2 v}\right)\end{array}$ & $\begin{array}{c}\text { Vacant Trigonal } \\
\text { Bipyramid } \\
\left(\boldsymbol{C}_{3 v}\right)\end{array}$ \\
\cline { 2 - 5 } & $\underline{1.332}$ & 28.758 & 16.371 & 28.602 \\
\hline $\begin{array}{c}\text { Rh2 } \\
\text { (4-coordinate) }\end{array}$ & $\begin{array}{c}\text { Square Planar } \\
\left(\boldsymbol{D}_{4 h}\right)\end{array}$ & $\begin{array}{c}\text { Tetrahedron } \\
\left(\boldsymbol{T}_{\boldsymbol{d}}\right)\end{array}$ & $\begin{array}{c}\text { Seesaw } \\
\left(\boldsymbol{C}_{2 v}\right)\end{array}$ & $\begin{array}{c}\text { Vacant Trigonal } \\
\text { Bipyramid } \\
\left(\boldsymbol{C}_{3 v}\right)\end{array}$ \\
\cline { 2 - 5 } & $\underline{1.350}$ & 24.569 & 12.255 & 25.567 \\
\hline
\end{tabular}

Table S8. SHAPE-calculated continuous shape measurements for $\mathrm{Rh} 1$ and $\mathrm{Rh} 2$ in $\mathbf{2}$ (the underlined value indicates the closest match to an idealized coordination geometry).

\begin{tabular}{|c|c|c|c|c|c|}
\hline $\begin{array}{c}\text { Rh1 } \\
\text { (4-coordinate) })\end{array}$ & $\begin{array}{c}\text { Square } \\
\text { Planar } \\
\left(\boldsymbol{D}_{4 h}\right)\end{array}$ & $\begin{array}{c}\text { Tetrahedron } \\
\left(\boldsymbol{T}_{\boldsymbol{d}}\right)\end{array}$ & $\begin{array}{c}\text { Seesaw } \\
\left(\boldsymbol{C}_{2 v}\right)\end{array}$ & \multicolumn{2}{|c|}{$\begin{array}{c}\text { Vacant Trigonal Bipyramid } \\
\left(\boldsymbol{C}_{3 v}\right)\end{array}$} \\
\cline { 2 - 6 } & $\underline{0.970}$ & 26.834 & 13.469 & \multicolumn{2}{|c|}{27.290} \\
\hline $\begin{array}{c}\text { Rh2 } \\
(5-\text { coordinate })\end{array}$ & $\begin{array}{c}\text { Pentagon } \\
\left(\boldsymbol{D}_{5 h}\right)\end{array}$ & $\begin{array}{c}\text { Vacant } \\
\text { octahedron } \\
\left(\boldsymbol{C}_{4 v}\right)\end{array}$ & $\begin{array}{c}\text { Trigonal } \\
\text { Bipyramid } \\
\left(\boldsymbol{D}_{3 h}\right)\end{array}$ & $\begin{array}{c}\text { Spherical } \\
\text { Square } \\
\text { Pyramid } \\
\left(\boldsymbol{C}_{4 v}\right)\end{array}$ & $\begin{array}{c}\text { Johnson } \\
\text { Trigonal } \\
\text { Bipyramid } \\
\left(\boldsymbol{D}_{3 h}\right)\end{array}$ \\
\cline { 2 - 6 } & 26.215 & 3.775 & 5.245 & $\underline{3.459}$ & 9.128 \\
\hline
\end{tabular}

Table S9. SHAPE-calculated continuous shape measurements for $\mathrm{Rh} 1$ and $\mathrm{Rh} 2$ in $\mathbf{3}$ (the underlined value indicates the closest match to an idealized coordination geometry).

\begin{tabular}{|c|c|c|c|c|c|}
\hline $\begin{array}{c}\text { Rh1 } \\
(5 \text {-coordinate })\end{array}$ & $\begin{array}{c}\text { Pentagon } \\
\left(\boldsymbol{D}_{5 h}\right)\end{array}$ & $\begin{array}{c}\text { Vacant } \\
\text { octahedron } \\
\left(\boldsymbol{C}_{4 v}\right)\end{array}$ & $\begin{array}{c}\text { Trigonal } \\
\text { Bipyramid } \\
\left(\boldsymbol{D}_{3 h}\right)\end{array}$ & $\begin{array}{c}\text { Spherical } \\
\text { Square } \\
\text { Pyramid } \\
\left(\boldsymbol{C}_{4 v}\right)\end{array}$ & $\begin{array}{c}\text { Johnson } \\
\text { Trigonal } \\
\text { Bipyramid } \\
\left(\boldsymbol{D}_{3 h}\right)\end{array}$ \\
\cline { 2 - 6 } & 23.331 & $\underline{3.370}$ & 6.774 & 3.460 & 10.103 \\
\hline \multirow{2}{*}{$\begin{array}{c}\text { Rh2 } \\
\text { (5-coordinate) }\end{array}$} & $\begin{array}{c}\text { Pentagon } \\
\left(\boldsymbol{D}_{5 h}\right)\end{array}$ & $\begin{array}{c}\text { Vacant } \\
\text { octahedron } \\
\left(\boldsymbol{C}_{4 v}\right)\end{array}$ & $\begin{array}{c}\text { Trigonal } \\
\text { Bipyramid } \\
\left(\boldsymbol{D}_{3 h}\right)\end{array}$ & $\begin{array}{c}\text { Spherical } \\
\text { Square } \\
\text { Pyramid } \\
\left(\boldsymbol{C}_{4 v}\right)\end{array}$ & $\begin{array}{c}\text { Johnson } \\
\text { Trigonal } \\
\text { Bipyramid } \\
\left(\boldsymbol{D}_{3 h}\right)\end{array}$ \\
\cline { 2 - 6 } & 22.728 & 3.676 & 7.316 & $\underline{3.612}$ & 10.695 \\
\hline
\end{tabular}




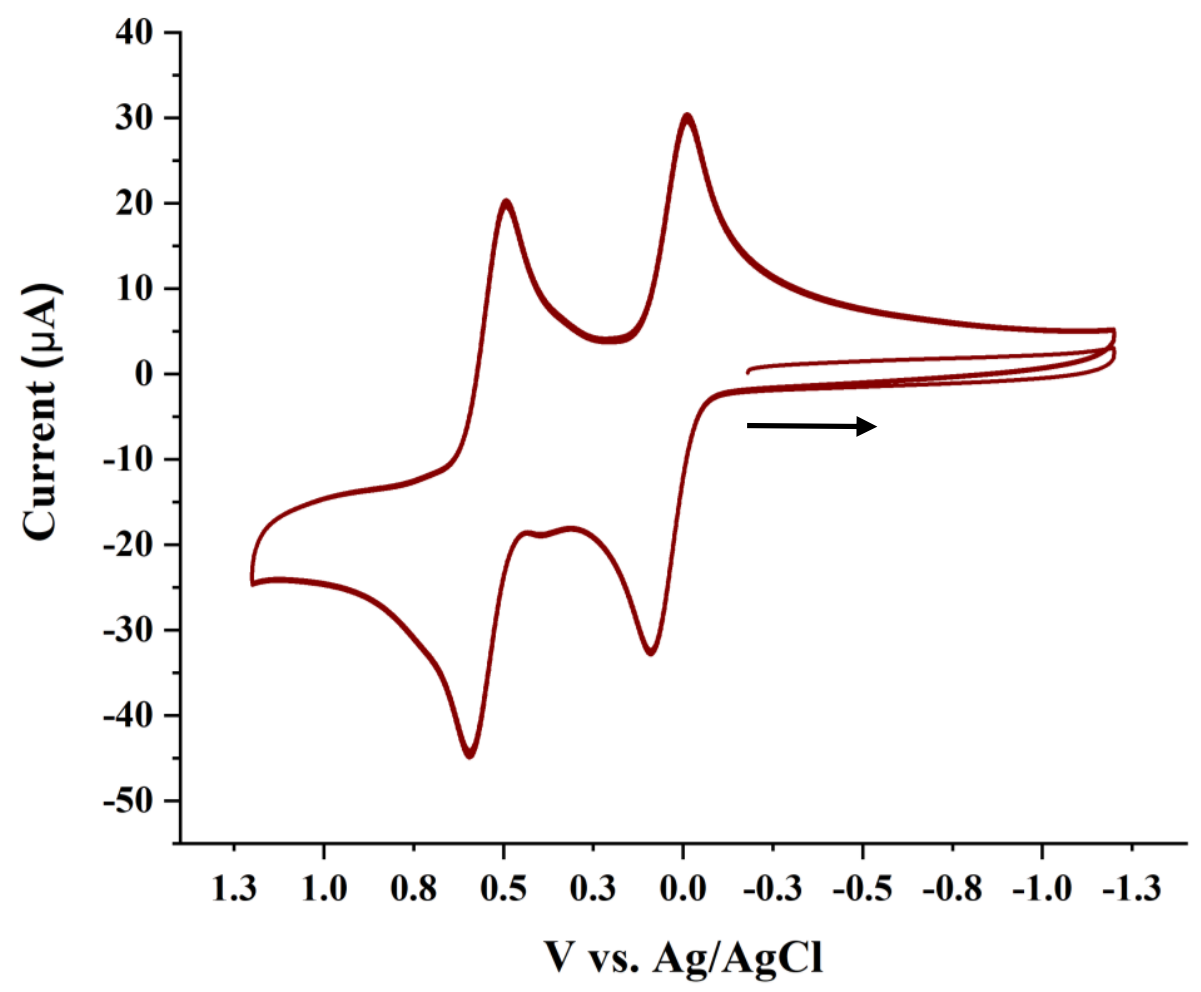

Figure S11. Cyclic voltammogram of $1(1 \mathrm{mM})$ over $\mu 10$ potential sweeps $(200 \mathrm{mV} / \mathrm{s})$.

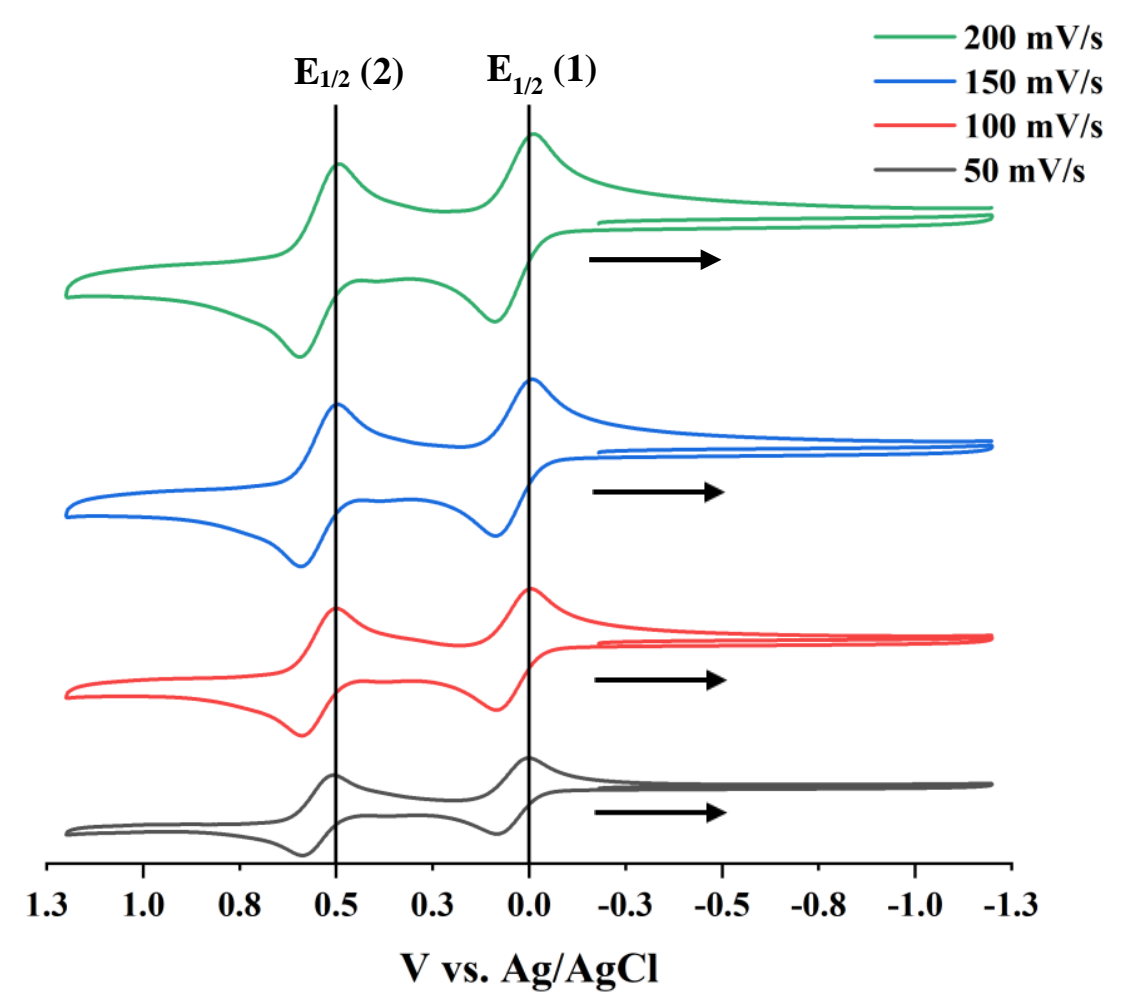

Figure S12. Cyclic voltammogram of $\mathbf{1}(1 \mathrm{mM})$ at various scan rates. 
Table S10. Electrochemical data collected from Figure S12.

$\begin{array}{ccccc}\text { Scan Rate }(\mathbf{m V} / \mathbf{s}) & \mathbf{E}_{\mathbf{1 / 2}}(\mathbf{1})(\mathbf{V} \text { vs. } \mathbf{A g} / \mathbf{A g C l}) & \boldsymbol{i}_{p, a} / i_{p, c}(\mathbf{1}) & \mathbf{E}_{1 / 2}(\mathbf{2})(\mathbf{V} \text { vs. } \mathbf{A g} / \mathbf{A g C l}) & \boldsymbol{i}_{p, a} / i_{p, c}(\mathbf{2}) \\ 100 & 0.042 & 1.00 & 0.55 & 0.87 \\ 150 & 0.040 & 1.08 & 0.54 & 0.88 \\ 200 & 0.039 & 1.12 & 0.54 & 0.89 \\ 250 & 0.038 & 1.13 & 0.54 & 0.90\end{array}$

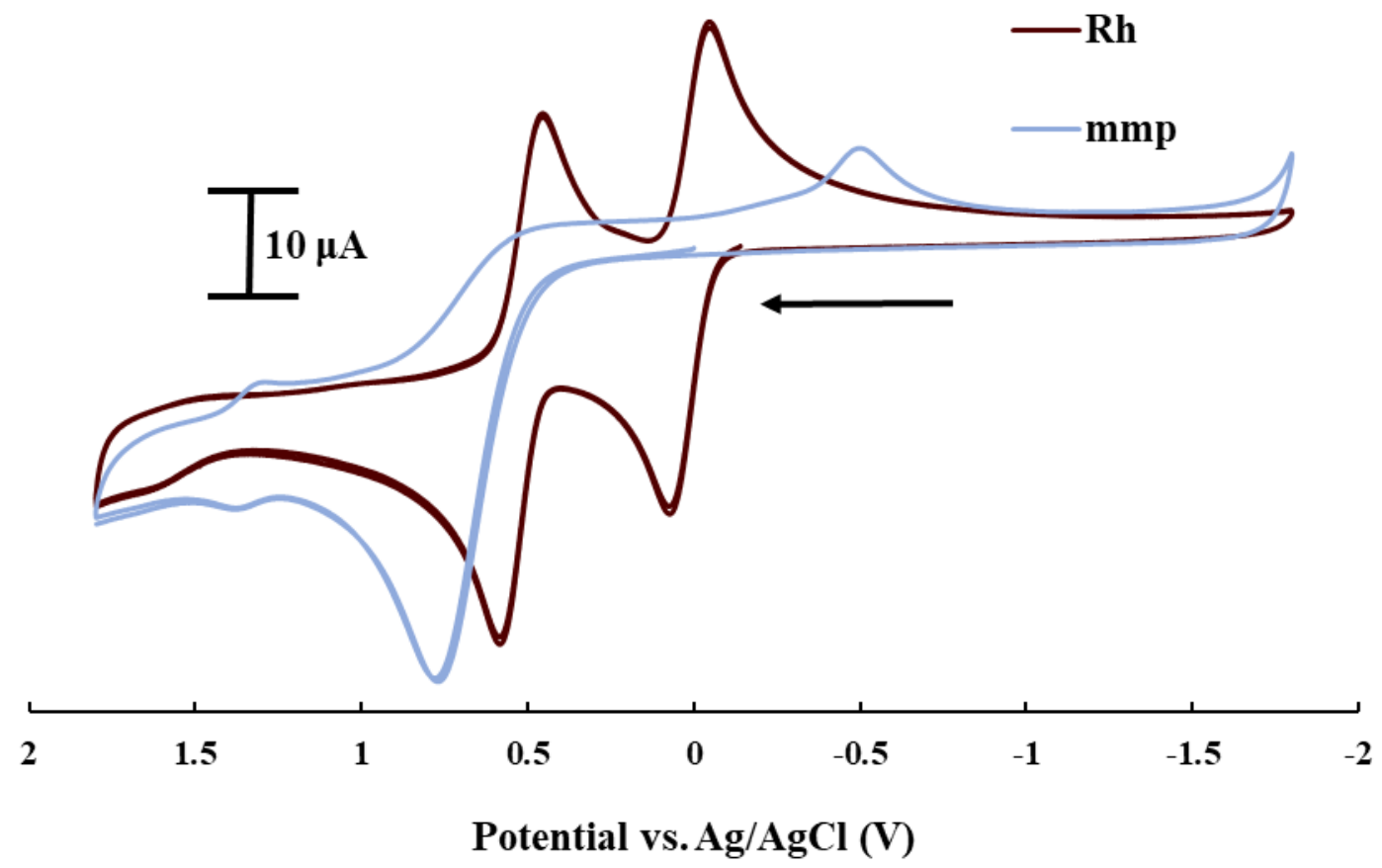

Figure S13. Cyclic voltammogram of $1(1 \mathrm{mM})$ and 2-mercapto-6-methylpyridine (mmp) (1 mM). 


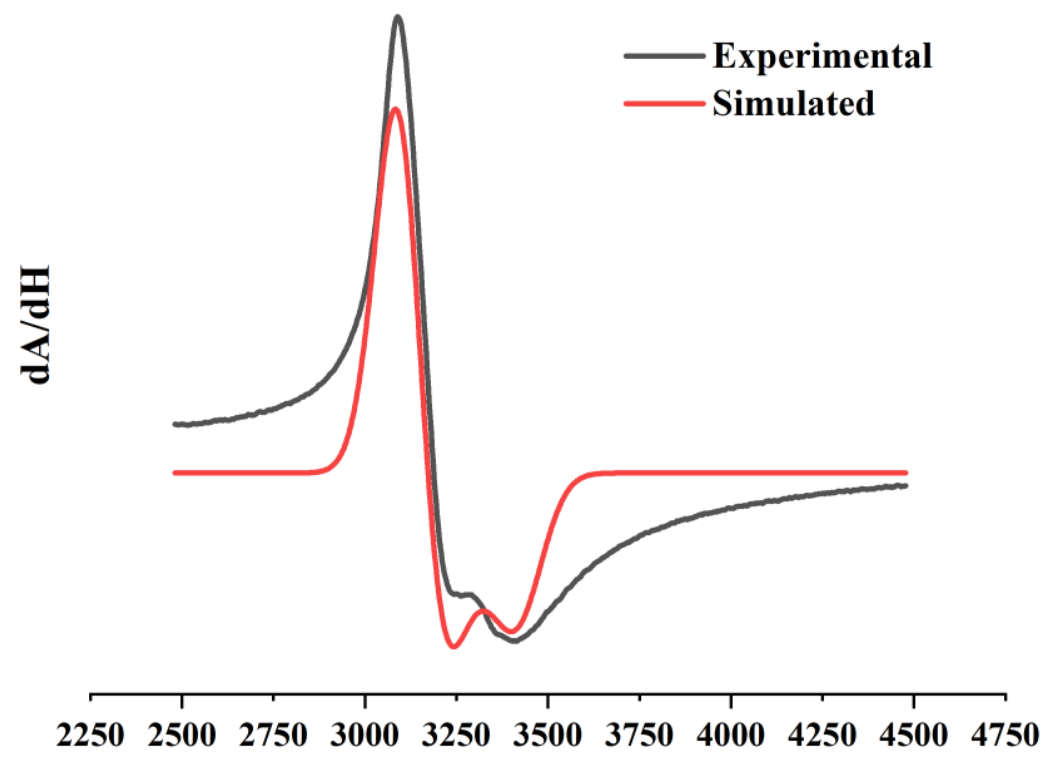

Magnetic Field (G)

Figure S14. EPR spectrum of 2 taken at $298 \mathrm{~K}$ (frequency $=9.38 \mathrm{GHz}, g_{\perp}=2.13, g_{\|}=1.97$, simulation linewidth=16).

Table S11. Hyperfine coupling parameters for simulating the 4 K EPR spectrum of 2.

$\begin{array}{ccc}\text { Nucleus } & \mathbf{A}_{\perp}(\mathbf{M H z}) & \mathbf{A}_{\|}(\mathbf{M H z}) \\ { }^{103} \mathrm{Rh} & 30,33.3 & 52,51.4 \\ { }^{14} \mathrm{~N} & 66.1,78.1 & 155,155.1\end{array}$
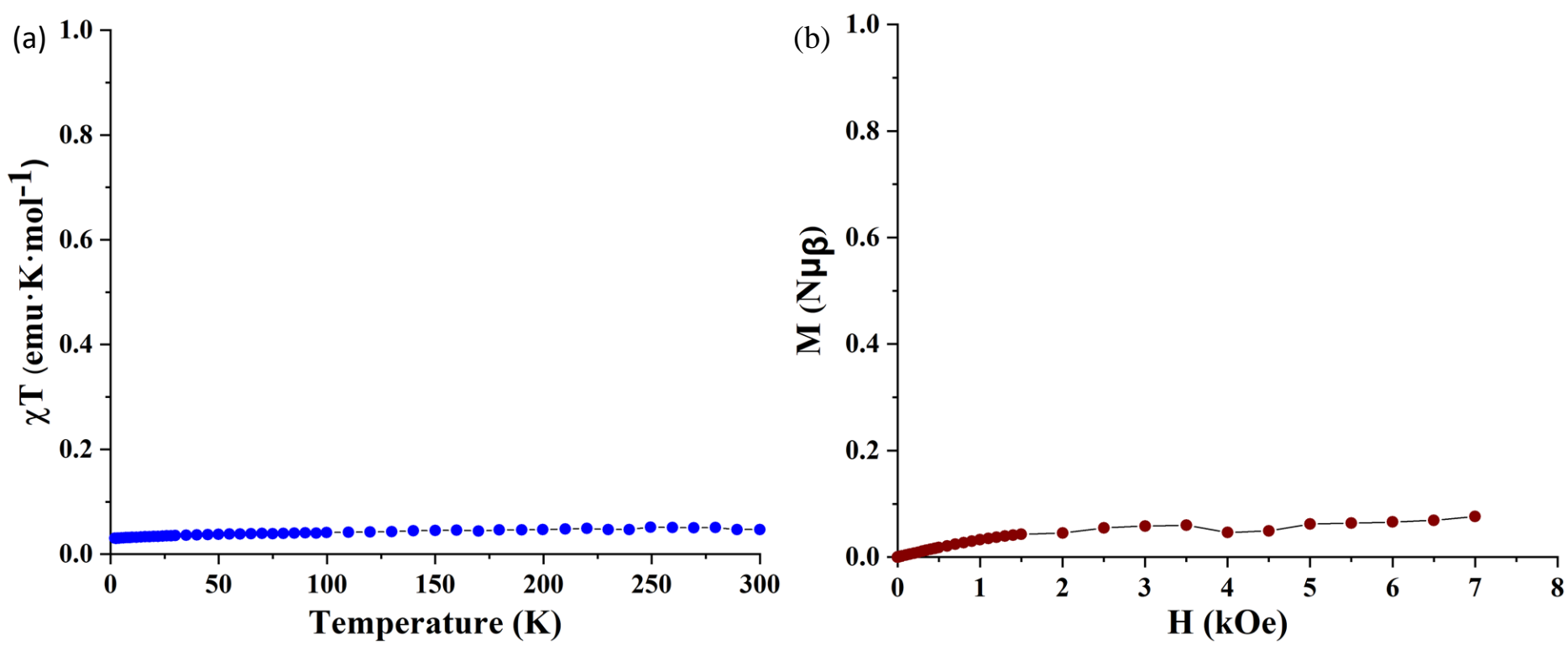

Figure S15. (a) $\chi \mathrm{T}$ vs. T plot of $\mathbf{3}$ from 2-300 K measured under an applied field of $1000 \mathrm{Oe}$, (b) Magnetization vs. field plot of 3 at $2 \mathrm{~K}$. 


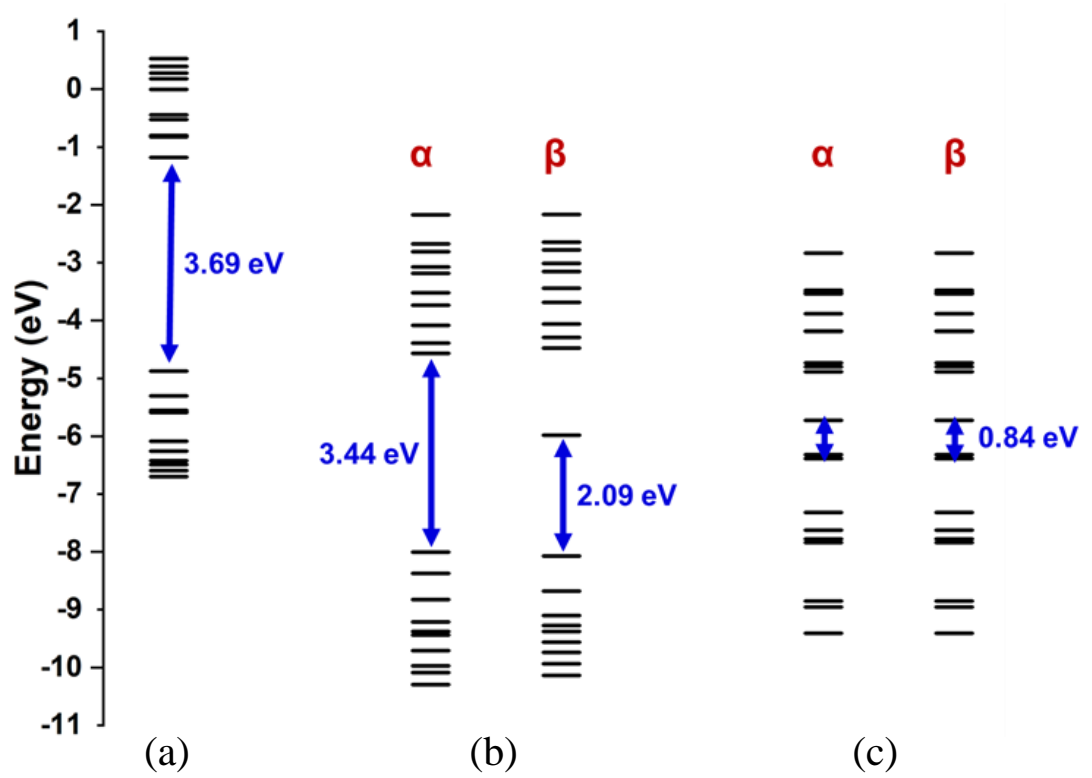

Figure S16. Frontier orbital diagrams of (a) 1, (b) $[2]^{+}$, and (c) $[3]^{2+}$.

Table S12. Frontier orbitals of 1 determined from single point calculations (isovalue=0.04).

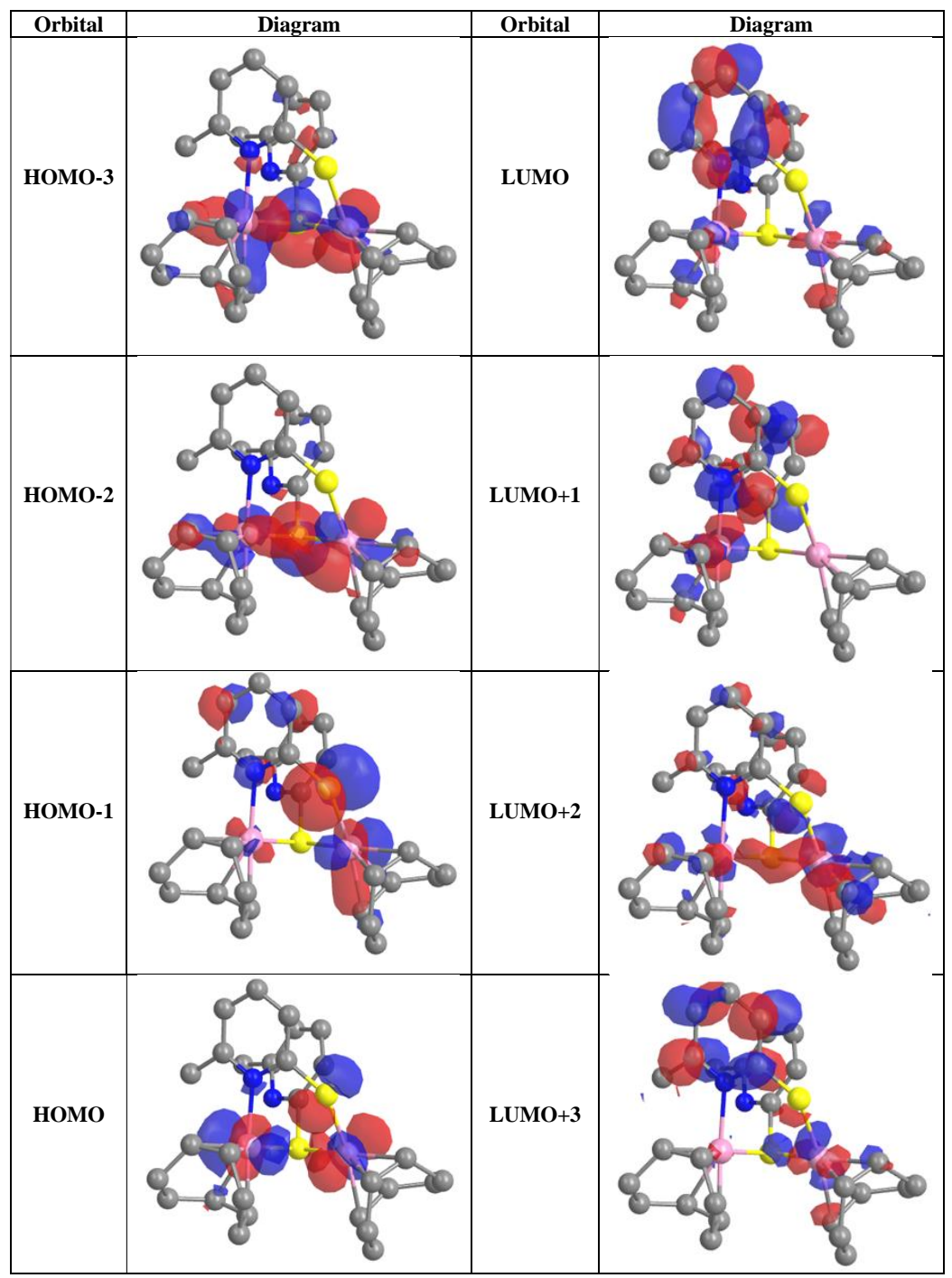


Table S13. Frontier $\alpha$ orbitals of [2] $]^{+}$determined from single point calculations (isovalue $=0.04$ ).

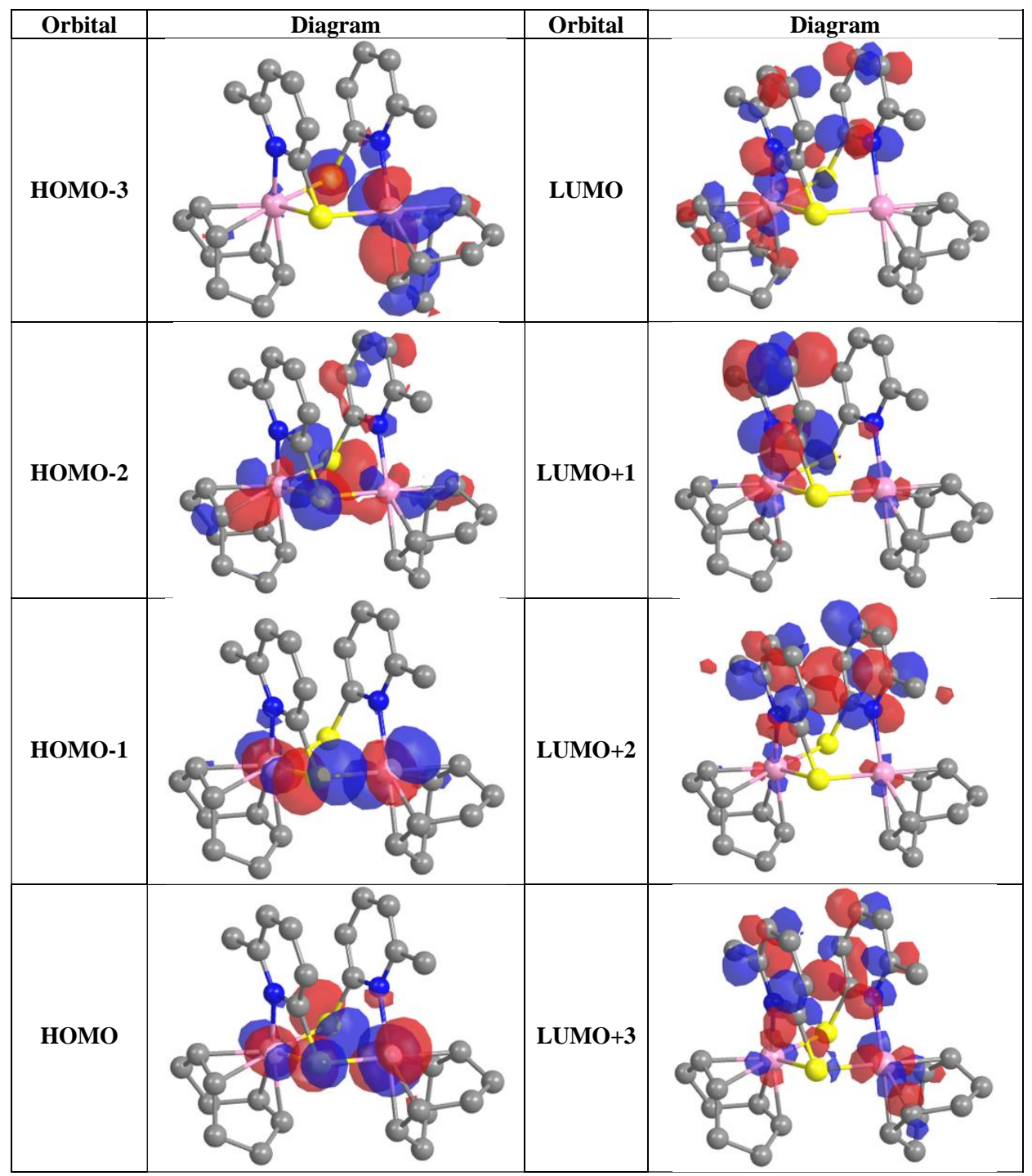


Table S14. Frontier $\beta$ orbitals of [2] $]^{+}$determined from single point calculations (isovalue $=0.04$ ).

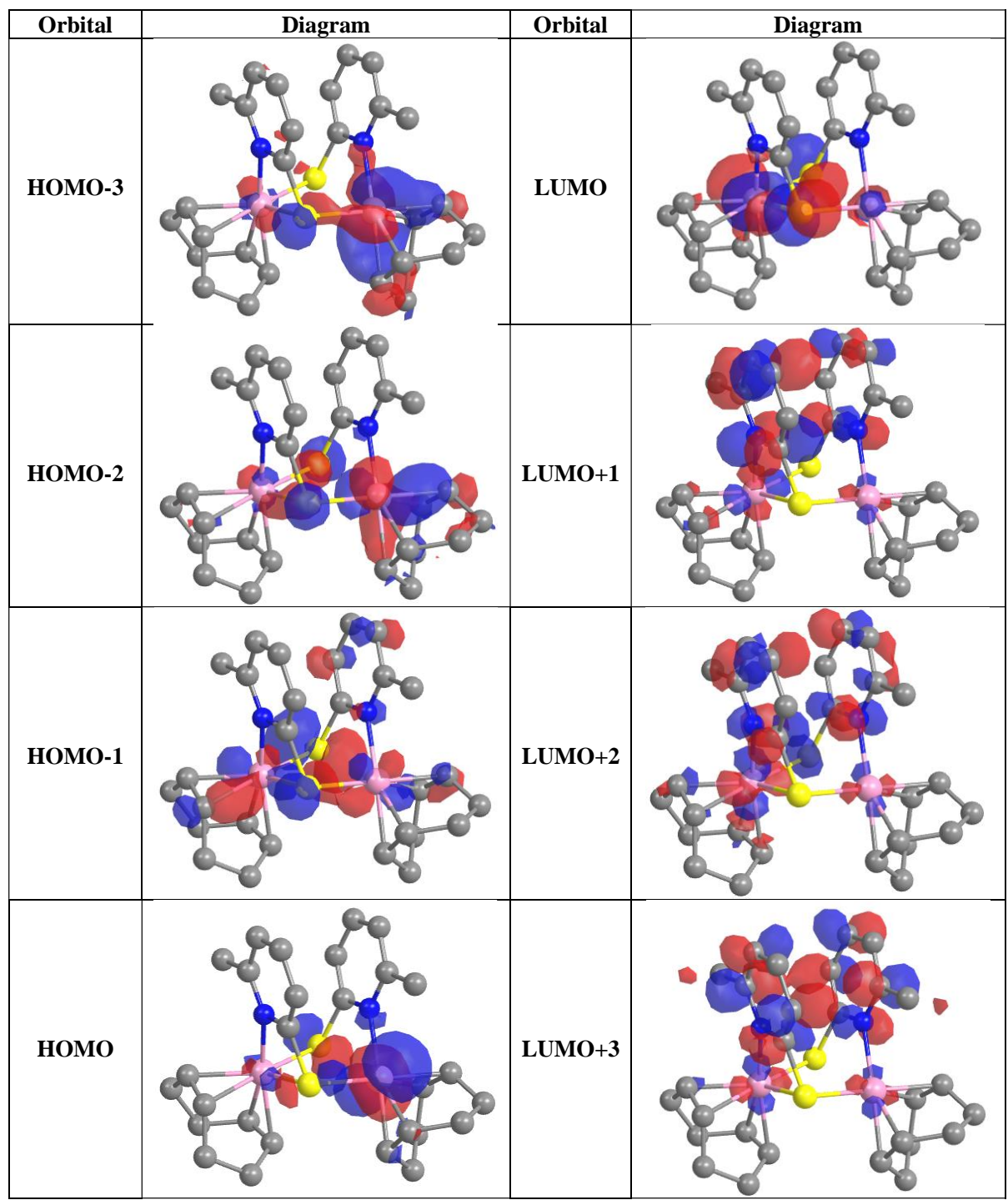


Table S15. Frontier $\alpha$ and $\beta$ orbitals of $[3]^{2+}$ determined from single point calculations (isovalue=0.04).

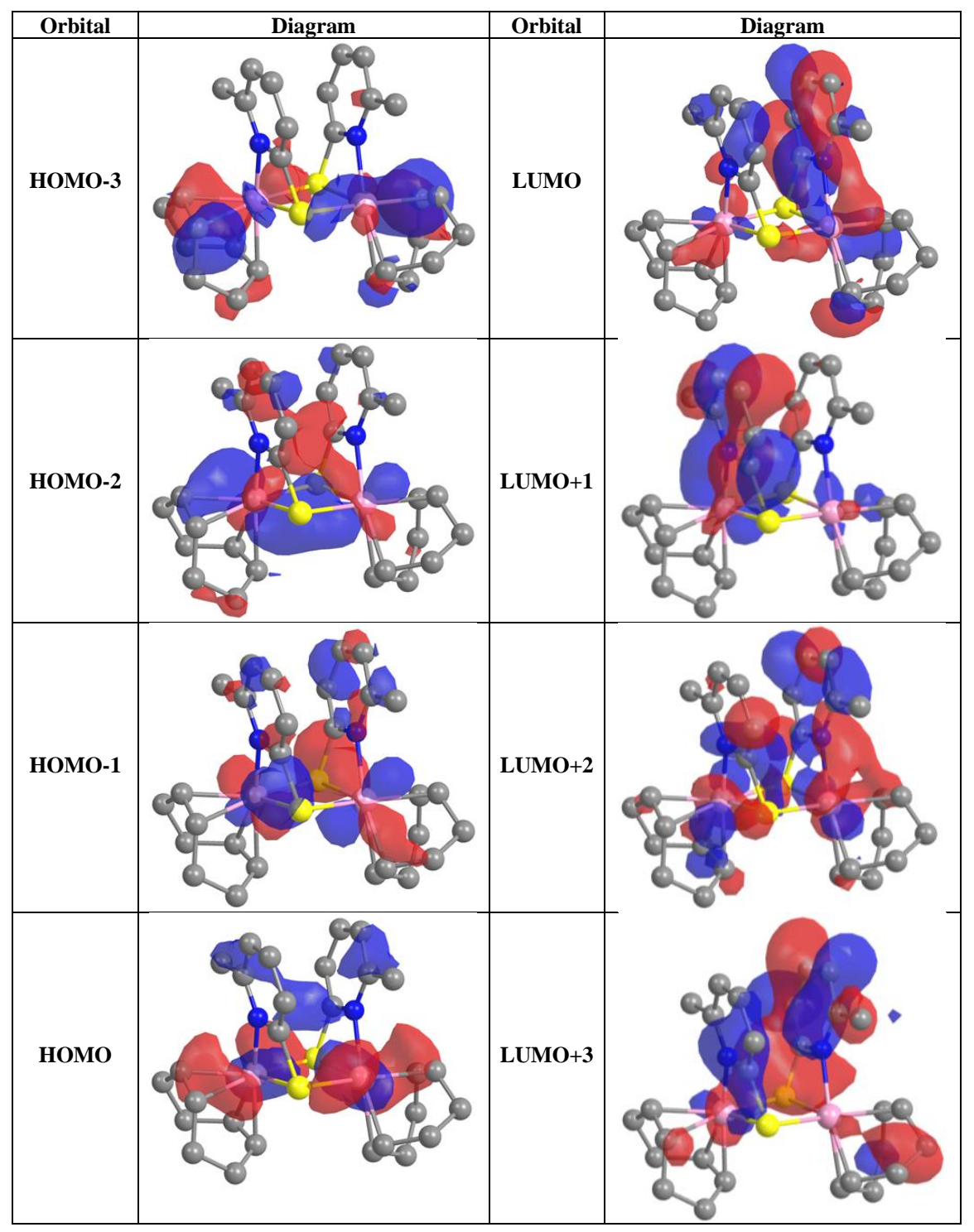

Table S16. Cartesian coordinates of gas-phase optimized 1.

$\begin{array}{cccc}\text { Symbol } & \mathbf{X} & \mathbf{Y} & \mathbf{Z} \\ \mathrm{Rh} & 4.65969 & 3.625419 & 5.845654 \\ \mathrm{Rh} & 5.565544 & 4.63072 & 2.904585 \\ \mathrm{~S} & 6.782069 & 3.711422 & 4.724133 \\ \mathrm{~S} & 3.911448 & 5.836983 & 5.363751 \\ \mathrm{~N} & 5.899256 & 6.585011 & 3.70849 \\ \mathrm{~N} & 9.003686 & 5.143319 & 4.899177 \\ \mathrm{C} & 7.489654 & 5.514239 & 6.727398 \\ \mathrm{H} & 6.52161 & 5.307539 & 7.173651 \\ \mathrm{C} & 5.087506 & 6.960289 & 4.718862 \\ \mathrm{C} & 4.873215 & 1.493895 & 5.97104\end{array}$




\begin{tabular}{|c|c|c|c|}
\hline $\mathrm{H}$ & 5.575008 & 1.189829 & 5.194122 \\
\hline $\mathrm{C}$ & 7.838955 & 4.922016 & 5.506494 \\
\hline & 5.442167 & 2.107732 & 7.105218 \\
\hline $\mathrm{H}$ & 6.528269 & 2.211751 & 7.102524 \\
\hline$c$ & 3.031504 & 3.513919 & 7.287084 \\
\hline $\mathrm{H}$ & 2.831268 & 4.545245 & 7.573628 \\
\hline $\mathrm{C}$ & 2.574284 & 3.112424 & 6.025371 \\
\hline $\mathrm{H}$ & 2.020797 & 3.850244 & 5.443998 \\
\hline $\mathrm{C}$ & 3.771393 & 5.160001 & 1.883148 \\
\hline $\mathrm{H}$ & 3.337158 & 5.981766 & 2.45242 \\
\hline $\mathrm{C}$ & 6.229187 & 3.105852 & 1.59375 \\
\hline $\mathrm{H}$ & 7.159454 & 2.717765 & 2.009924 \\
\hline $\mathrm{C}$ & 9.889689 & 5.963383 & 5.478099 \\
\hline $\mathrm{C}$ & 4.82099 & 2.188598 & 8.480173 \\
\hline $\mathrm{H}$ & 4.970489 & 1.246104 & 9.031132 \\
\hline $\mathrm{H}$ & 5.36551 & 2.958044 & 9.041268 \\
\hline $\mathrm{C}$ & 2.378665 & 1.684329 & 5.570786 \\
\hline $\mathrm{H}$ & 2.290846 & 1.698891 & 4.478112 \\
\hline $\mathrm{H}$ & 1.42073 & 1.290573 & 5.947454 \\
\hline $\mathrm{C}$ & 6.078715 & 9.151513 & 4.738535 \\
\hline $\mathrm{H}$ & 6.140911 & 10.16373 & 5.130188 \\
\hline $\mathrm{C}$ & 4.852819 & 5.467685 & 1.04247 \\
\hline $\mathrm{H}$ & 5.157575 & 6.513908 & 1.02907 \\
\hline $\mathrm{C}$ & 5.150229 & 8.262142 & 5.244858 \\
\hline $\mathrm{H}$ & 4.47222 & 8.534788 & 6.045976 \\
\hline $\mathrm{C}$ & 3.334059 & 2.565546 & 8.433246 \\
\hline $\mathrm{H}$ & 2.70824 & 1.670389 & 8.350414 \\
\hline $\mathrm{H}$ & 3.047946 & 3.043564 & 9.377198 \\
\hline $\mathrm{C}$ & 8.411137 & 6.364732 & 7.32326 \\
\hline $\mathrm{H}$ & 8.178398 & 6.844449 & 8.270962 \\
\hline $\mathrm{C}$ & 5.035138 & 2.677947 & 2.207965 \\
\hline $\mathrm{H}$ & 5.141604 & 1.992569 & 3.047467 \\
\hline $\mathrm{C}$ & 9.633772 & 6.592776 & 6.697693 \\
\hline $\mathrm{H}$ & 10.37798 & 7.246279 & 7.143285 \\
\hline $\mathrm{C}$ & 6.955421 & 8.725798 & 3.740828 \\
\hline $\mathrm{H}$ & 7.721341 & 9.386218 & 3.347665 \\
\hline $\mathrm{C}$ & 6.853037 & 7.429081 & 3.250731 \\
\hline C & 6.358206 & 3.631931 & 0.182263 \\
\hline $\mathrm{H}$ & 6.369012 & 2.799051 & -0.53933 \\
\hline $\mathrm{H}$ & 7.339252 & 4.115683 & 0.101941 \\
\hline $\mathrm{C}$ & 3.541443 & 0.76028 & 5.964521 \\
\hline $\mathrm{H}$ & 3.361646 & 0.303627 & 6.944347 \\
\hline $\mathrm{H}$ & 3.593463 & -0.07407 & 5.255537 \\
\hline $\mathrm{C}$ & 7.819977 & 6.908043 & 2.231196 \\
\hline $\mathrm{H}$ & 8.352586 & 6.041289 & 2.641483 \\
\hline
\end{tabular}




$\begin{array}{cccc}\mathrm{H} & 8.549045 & 7.679147 & 1.963115 \\ \mathrm{H} & 7.310747 & 6.575877 & 1.322046 \\ \mathrm{C} & 3.677535 & 2.648469 & 1.523103 \\ \mathrm{H} & 3.102767 & 1.794057 & 1.898968 \\ \mathrm{H} & 3.801586 & 2.469128 & 0.449021 \\ \mathrm{C} & 5.266307 & 4.65165 & -0.17132 \\ \mathrm{H} & 5.635923 & 5.327516 & -0.95157 \\ \mathrm{H} & 4.390566 & 4.154234 & -0.602 \\ \mathrm{C} & 11.18165 & 6.171398 & 4.738014 \\ \mathrm{H} & 11.65188 & 5.207618 & 4.516342 \\ \mathrm{H} & 11.88464 & 6.782861 & 5.311395 \\ \mathrm{H} & 10.99753 & 6.666305 & 3.777263 \\ \mathrm{C} & 2.886063 & 3.940949 & 1.775924 \\ \mathrm{H} & 2.117211 & 4.08998 & 1.000621 \\ \mathrm{H} & 2.35106 & 3.850483 & 2.728016\end{array}$

Table S17. Cartesian coordinates of the gas-phase optimized structure of [2] ${ }^{+}$.

$\begin{array}{cccc}\text { Symbol } & \mathbf{X} & \mathbf{Y} & \mathbf{Z} \\ \mathrm{Rh} & 3.742361 & 11.37701 & 6.937605 \\ \mathrm{Rh} & 5.41689 & 9.947003 & 4.007667 \\ \mathrm{~S} & 3.002087 & 10.20166 & 4.984669 \\ \mathrm{~S} & 6.450515 & 11.52066 & 5.403183 \\ \mathrm{~N} & 4.33566 & 13.10632 & 5.79576 \\ \mathrm{~N} & 4.238339 & 11.38788 & 3.034826 \\ \mathrm{C} & 1.943519 & 12.06113 & 3.157715 \\ \mathrm{H} & 0.976851 & 11.96629 & 3.64001 \\ \mathrm{C} & 2.151564 & 12.90531 & 2.072531 \\ \mathrm{H} & 1.331207 & 13.49647 & 1.675079 \\ \mathrm{C} & 3.614405 & 14.24178 & 5.680353 \\ \mathrm{C} & 3.41844 & 12.99774 & 1.502928 \\ \mathrm{H} & 3.605436 & 13.66489 & 0.667686 \\ \mathrm{C} & 6.184233 & 14.16227 & 4.686765 \\ \mathrm{H} & 7.195318 & 14.0799 & 4.303438 \\ \mathrm{C} & 4.464129 & 12.22068 & 2.001143 \\ \mathrm{C} & 5.581168 & 13.04886 & 5.273765 \\ \mathrm{C} & 4.163792 & 15.38167 & 5.092934 \\ \mathrm{H} & 3.564919 & 16.28455 & 5.025966 \\ \mathrm{C} & 5.466704 & 15.34926 & 4.611722 \\ \mathrm{H} & 5.913305 & 16.23514 & 4.168733 \\ \mathrm{C} & 2.203625 & 14.256 & 6.190138 \\ \mathrm{H} & 1.886038 & 13.24925 & 6.477577 \\ \mathrm{H} & 1.525819 & 14.64246 & 5.421256 \\ \mathrm{H} & 2.105184 & 14.91201 & 7.063199 \\ \mathrm{C} & 3.027532 & 11.32495 & 3.624701\end{array}$




\begin{tabular}{|c|c|c|c|}
\hline $\mathrm{C}$ & 2.335194 & 10.23455 & 8.079203 \\
\hline H & 1.610802 & 9.822848 & 7.375771 \\
\hline & 4.996626 & 11.98903 & 8.56418 \\
\hline $\mathrm{H}$ & 5.761859 & 12.63052 & 8.126666 \\
\hline & 3.586967 & 9.599354 & 8.137548 \\
\hline & 3.717968 & 8.74636 & 7.471184 \\
\hline 0 & 4.513931 & 9.622071 & 9.343327 \\
\hline & 5.045324 & 8.666268 & 9.405105 \\
\hline $\mathrm{H}$ & 3.921106 & 9.687544 & 10.26157 \\
\hline & 3.729786 & 12.55734 & 8.758147 \\
\hline 5 & 3.616632 & 13.59374 & 8.443259 \\
\hline 0 & 5.536171 & 10.76611 & 9.265305 \\
\hline & 5.90071 & 11.03392 & 10.2686 \\
\hline $\mathrm{H}$ & 6.414526 & 10.43048 & 8.702626 \\
\hline 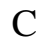 & 4.526533 & 8.256605 & 2.852609 \\
\hline $\mathrm{H}$ & 3.535446 & 8.603 & \\
\hline C & 7.524736 & 7.725458 & 3.418147 \\
\hline $\mathrm{H}$ & 7.232426 & 6.681327 & 3.563143 \\
\hline $\mathrm{H}$ & 8.61934 & 7.732347 & 3.407045 \\
\hline 0 & 5.916185 & 8.27787 & 5.356982 \\
\hline $\mathrm{H}$ & 5.868702 & 8.728 & 6.34 \\
\hline C & 5.598814 & 8.805124 & 2.149851 \\
\hline $\mathrm{H}$ & 5.343527 & 9.503774 & 1.353587 \\
\hline $\mathrm{C}$ & 4.566784 & 6.956816 & 3.634001 \\
\hline $\mathrm{H}$ & 5.25 & 6.260072 & 3.145281 \\
\hline $\mathrm{H}$ & 3.579823 & 6.486982 & 3.5805 \\
\hline $\mathrm{C}$ & 7.048417 & 8.553524 & 4.597424 \\
\hline $\mathrm{H}$ & 7.796441 & 9.203287 & 5.0 \\
\hline $\mathrm{C}$ & 4.940795 & 7.152841 & 5.112009 \\
\hline $\mathrm{H}$ & 4.029504 & 7.370507 & 5.678248 \\
\hline $\mathrm{H}$ & 5.346904 & 6.222185 & 5.535685 \\
\hline $\mathrm{C}$ & 7.005905 & 8.260201 & 2.077044 \\
\hline $\mathrm{H}$ & 7.661838 & 9.070077 & 1.737156 \\
\hline $\mathrm{H}$ & 7.060274 & 7.482367 & 1.300494 \\
\hline $\mathrm{C}$ & 1.716853 & 11.0906 & 9.158149 \\
\hline $\mathrm{H}$ & 0.892808 & 11.65239 & 8.702604 \\
\hline $\mathrm{H}$ & 1.257989 & 10.45155 & 9.928027 \\
\hline $\mathrm{C}$ & 2.715654 & 12.07344 & 9.779399 \\
\hline $\mathrm{H}$ & 2.175835 & 12.93529 & 10.1859 \\
\hline $\mathrm{H}$ & 3.235815 & 11.62047 & 10.62928 \\
\hline $\mathrm{C}$ & 5.841706 & 12.27928 & 1.415663 \\
\hline $\mathrm{H}$ & 6.589157 & 11.95933 & 2.147481 \\
\hline $\mathrm{H}$ & 6.07786 & 13.29606 & 1.08883 \\
\hline & 5.924281 & 11.63102 & 0.53444 \\
\hline
\end{tabular}


Table S18. Cartesian coordinates of the gas-phase optimized structure of $[3]^{2+}$.

$\begin{array}{cccc}\text { Symbol } & \mathbf{X} & \mathbf{Y} & \mathbf{Z} \\ \mathrm{Rh} & 3.800149 & 22.69097 & -6.43551 \\ \mathrm{Rh} & 2.551868 & 23.0651 & -3.31484 \\ \mathrm{~S} & 4.738825 & 23.02191 & -4.07207 \\ \mathrm{~S} & 1.649214 & 22.4097 & -5.59762 \\ \mathrm{~N} & 2.233005 & 21.00704 & -3.51807 \\ \mathrm{~N} & 4.562648 & 20.9371 & -5.57943 \\ \mathrm{C} & 5.937592 & 20.49951 & -3.65842 \\ \mathrm{H} & 6.403043 & 20.86335 & -2.74854 \\ \mathrm{C} & 1.068106 & 19.68095 & -5.14675 \\ \mathrm{H} & 0.565175 & 19.62086 & -6.10609 \\ \mathrm{C} & 0.41123 & 23.41189 & -2.73906 \\ \mathrm{H} & -0.13928 & 22.61989 & -3.2453 \\ \mathrm{C} & 2.409056 & 25.28322 & -3.61406 \\ \mathrm{H} & 3.114577 & 25.50432 & -4.41192 \\ \mathrm{C} & 6.127038 & 19.19647 & -4.1214 \\ \mathrm{H} & 6.75956 & 18.50816 & -3.56796 \\ \mathrm{C} & 1.169189 & 18.58472 & -4.28933 \\ \mathrm{H} & 0.733435 & 17.63042 & -4.57142 \\ \mathrm{C} & 2.968603 & 25.09478 & -2.35986 \\ \mathrm{H} & 4.055856 & 25.11951 & -2.30191 \\ \mathrm{C} & 3.068992 & 20.13068 & -1.38194 \\ \mathrm{H} & 2.363626 & 20.05826 & -0.54523 \\ \mathrm{H} & 3.572602 & 21.09948 & -1.33001 \\ \mathrm{H} & 3.810924 & 19.33935 & -1.23504 \\ \mathrm{C} & 4.036333 & 19.25819 & -7.29416 \\ \mathrm{H} & 4.772301 & 19.0652 & -8.08398 \\ \mathrm{H} & 3.339738 & 20.02403 & -7.64471 \\ \mathrm{H} & 3.486454 & 18.32342 & -7.14454 \\ \mathrm{C} & -0.03877 & 24.82572 & -3.0565 \\ \mathrm{H} & 1.44853 & 25.9846 & -1.14482 \\ \mathrm{H} & -0.25337 & 25.35408 & -2.12313 \\ \mathrm{H} & -0.99085 & 24.77407 & -3.59268 \\ \mathrm{C} & 5.499248 & 18.78199 & -5.2898 \\ \mathrm{H} & 5.624797 & 17.76755 & -5.65517 \\ \mathrm{C} & 2.366319 & 19.95167 & -2.69153 \\ \mathrm{C} & 1.133271 & 23.02207 & -1.6131 \\ \mathrm{H} & 1.054074 & 21.97133 & -1.33777 \\ \mathrm{C} & 1.634274 & 20.86843 & -4.71547 \\ \mathrm{C} & 1.834059 & 18.71676 & -3.0756 \\ \mathrm{H} & 4.933347 & 17.86935 & -2.40449 \\ \mathrm{H} & 2.711365 & 19.67474 & -6.02441 \\ \mathrm{H} & & & \\ \mathrm{H} & & \end{array}$




$\begin{array}{llll}\mathrm{H} & 2.941584 & 25.64106 & -0.30321 \\ \mathrm{C} & 5.130673 & 21.32574 & -4.4215 \\ \mathrm{C} & 1.680498 & 23.90834 & -0.52512 \\ \mathrm{H} & 2.471818 & 23.35841 & -0.00317 \\ \mathrm{H} & 0.895221 & 24.08247 & 0.225516 \\ \mathrm{C} & 5.227092 & 22.49563 & -8.13754 \\ \mathrm{H} & 5.397987 & 21.42229 & -8.19607 \\ \mathrm{C} & 3.555911 & 24.84449 & -7.09988 \\ \mathrm{H} & 2.987627 & 25.32509 & -6.30803 \\ \mathrm{C} & 6.102679 & 24.65137 & -7.00604 \\ \mathrm{H} & 6.987275 & 24.92203 & -7.6003 \\ \mathrm{H} & 6.358751 & 24.88355 & -5.96738 \\ \mathrm{C} & 5.875507 & 23.16566 & -7.10295 \\ \mathrm{H} & 6.523406 & 22.56313 & -6.46614 \\ \mathrm{C} & 4.695608 & 23.14122 & -9.40258 \\ \mathrm{H} & 4.830186 & 22.44282 & -10.2343 \\ \mathrm{H} & 5.30179 & 24.01765 & -9.64716 \\ \mathrm{C} & 3.21184 & 23.50755 & -9.2963 \\ \mathrm{H} & 2.934123 & 24.24718 & -10.0629 \\ \mathrm{H} & 2.597946 & 22.62417 & -9.50653 \\ \mathrm{C} & 2.806718 & 24.04249 & -7.94868 \\ \mathrm{H} & 1.736211 & 23.98887 & -7.7532 \\ \mathrm{C} & 0.968042 & 25.60993 & -3.91188 \\ \mathrm{H} & 0.782094 & 25.39395 & -4.9699 \\ \mathrm{H} & 0.812919 & 26.69243 & -3.79625 \\ \mathrm{C} & 4.890198 & 25.48736 & -7.43448 \\ \mathrm{H} & 4.936327 & 26.46182 & -6.93843 \\ \mathrm{H} & 4.919697 & 25.70297 & -8.50637\end{array}$




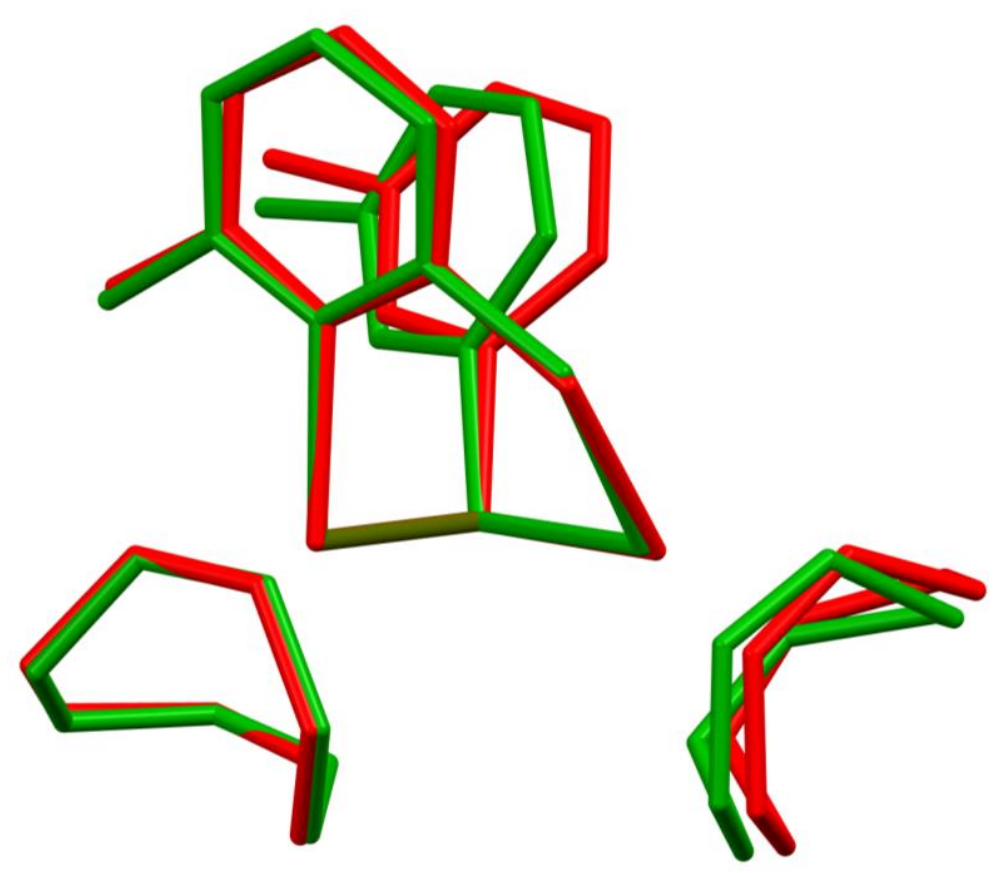

Figure S17. Structural overlay of the crystal structure of $\mathbf{1}$ (green) with gas-phase optimized structure (red).

Table S19. Selected distances and bond angles for crystal and gas-phase optimized structures of $\mathbf{1}$.

$\begin{array}{ccc}\text { Distance } & \text { Crystal }(\AA) & \text { Optimized }(\AA) \\ \text { Rh1-S3 } & 2.36830 & 2.40202 \\ \text { Rh1-S4 } & 2.34709 & 2.38393 \\ \text { Rh2-S3 } & 2.34516 & 2.37398 \\ \text { Rh2-N5 } & 2.12848 & 2.13936 \\ \text { Angle } & \text { Crystal }\left(^{\circ}\right) & \text { Optimized }\left(^{\circ}\right) \\ \text { N5-Rh2-S3 } & 90.591 & 89.186 \\ \text { Rh1-S3-Rh2 } & 82.097 & 85.351 \\ \text { S4-Rh1-S3 } & 100.946 & 98.611\end{array}$




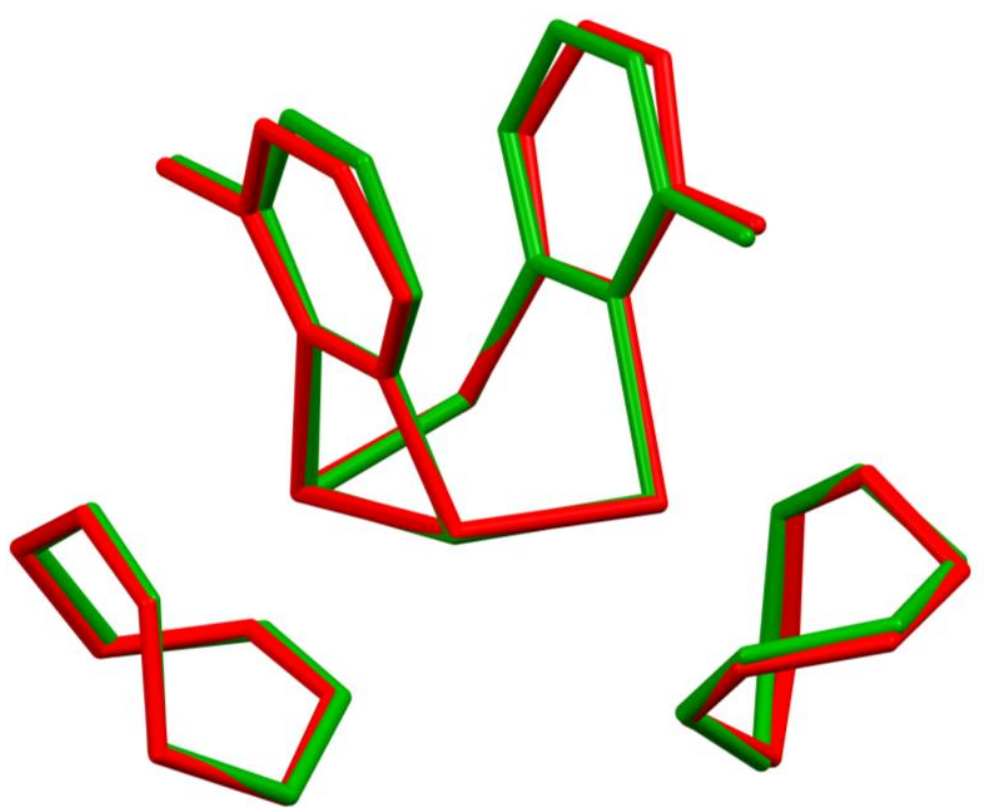

Figure S18. Structural overlay of the crystal structure of 2 (green) with gas-phase optimized structure (red).

Table S20. Selected distances and bond angles for crystal and gas-phase optimized structures of 2.

$\begin{array}{ccc}\text { Distance } & \text { Crystal }(\AA) & \text { Optimized }(\AA) \\ \text { Rh1-S3 } & 2.36029 & 2.39654 \\ \text { Rh1-S4 } & 3.08859 & 3.11596 \\ \text { Rh1-N5 } & 2.11361 & 2.15554 \\ \text { Rh2-S3 } & 2.56633 & 2.61738 \\ \text { Rh2-S4 } & 2.3362 & 2.34356 \\ \text { Rh2-N6 } & 2.07415 & 2.10036 \\ \text { Angle } & \text { Crystal }\left(^{\circ}\right) & \text { Optimized }\left(^{\circ}\right) \\ \text { N5-Rh1-S3 } & 93.353 & 92.683 \\ \text { Rh1-S3-Rh2 } & 91.935 & 93.837 \\ \text { N6-Rh2-S3 } & 66.658 & 65.698 \\ \text { N6-Rh2-S4 } & 93.313 & 93.592\end{array}$




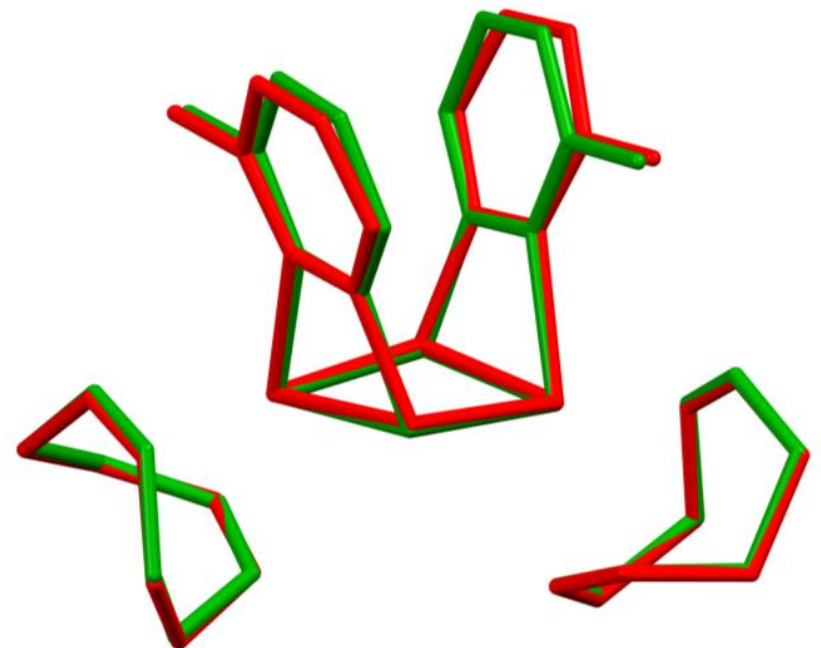

Figure S19. Structural overlay of the crystal structure of $\mathbf{3}$ (green) with gas-phase optimized structure (red).

Table S21. Selected distances and bond angles for crystal and gas-phase optimized structures of $\mathbf{3}$.

$\begin{array}{ccc}\text { Distance } & \text { Crystal }(\AA) & \text { Optimized }(\AA) \\ \text { Rh1-S3 } & 2.53809 & 2.56447 \\ \text { Rh1-S4 } & 2.32520 & 2.32544 \\ \text { Rh1-N6 } & 2.07425 & 2.09531 \\ \text { Rh2-S3 } & 2.31068 & 2.31474 \\ \text { Rh2-S4 } & 2.51966 & 2.54075 \\ \text { Rh2-N5 } & 2.07542 & 2.09251 \\ \text { Angle } & \text { Crystal }\left(^{\circ}\right) & \text { Optimized }\left(^{\circ}\right) \\ \text { N6-Rh1-S3 } & 66.888 & 66.314 \\ \text { N6-Rh1-S4 } & 95.154 & 95.057 \\ \text { Rh1-S3-Rh2 } & 86.800 & 87.597 \\ \text { Rh1-S4-Rh2 } & 86.923 & 87.932 \\ \text { N5-Rh2-S3 } & 95.269 & 95.385 \\ \text { N5-Rh2-S4 } & 67.014 & 66.727\end{array}$




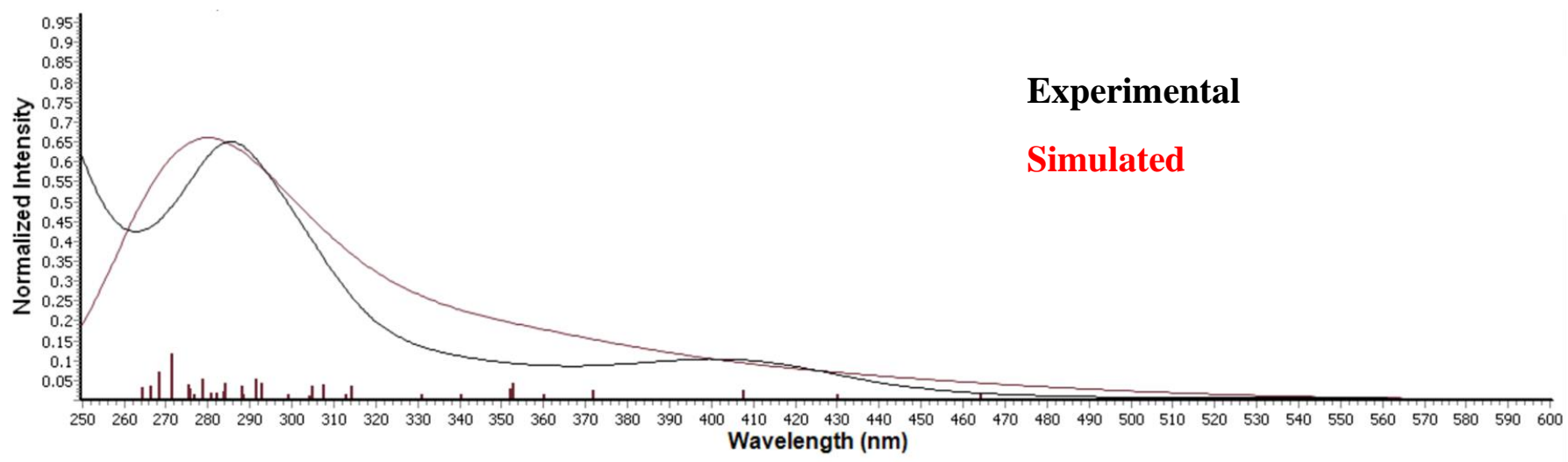

Figure S20. Experimental and TD-DFT calculated electronic absorption spectrum of $\mathbf{1}$.

Table S22. TD-DFT calculated excited states of $\mathbf{1 .}$

\begin{tabular}{|c|c|c|c|}
\hline$\lambda_{\exp }(\mathbf{n m})$ & $\lambda_{\text {calcd }}(\mathbf{n m})$ & $f$ & Major Contributions \\
\hline 285.5 & 284.2 & 0.043 & $\begin{array}{c}\mathrm{HOMO} \rightarrow \mathrm{LUMO}+8(29 \%) \\
\mathrm{HOMO}-2 \rightarrow \mathrm{LUMO}+5(26 \%) \\
\mathrm{HOMO}-2 \rightarrow \text { LUMO+3(19\%) } \\
\mathrm{HOMO}-5 \rightarrow \text { LUMO }(19 \%)\end{array}$ \\
\hline 399.5 & 407.6 & 0.026 & $\begin{array}{c}\mathrm{HOMO} \rightarrow \text { LUMO+1 }(85 \%) \\
\text { HOMO } \rightarrow \text { LUMO }(58 \%)\end{array}$ \\
\hline
\end{tabular}

Table S23. NTO's of TD-DFT calculated excited states of 1 (isovalue=0.04).

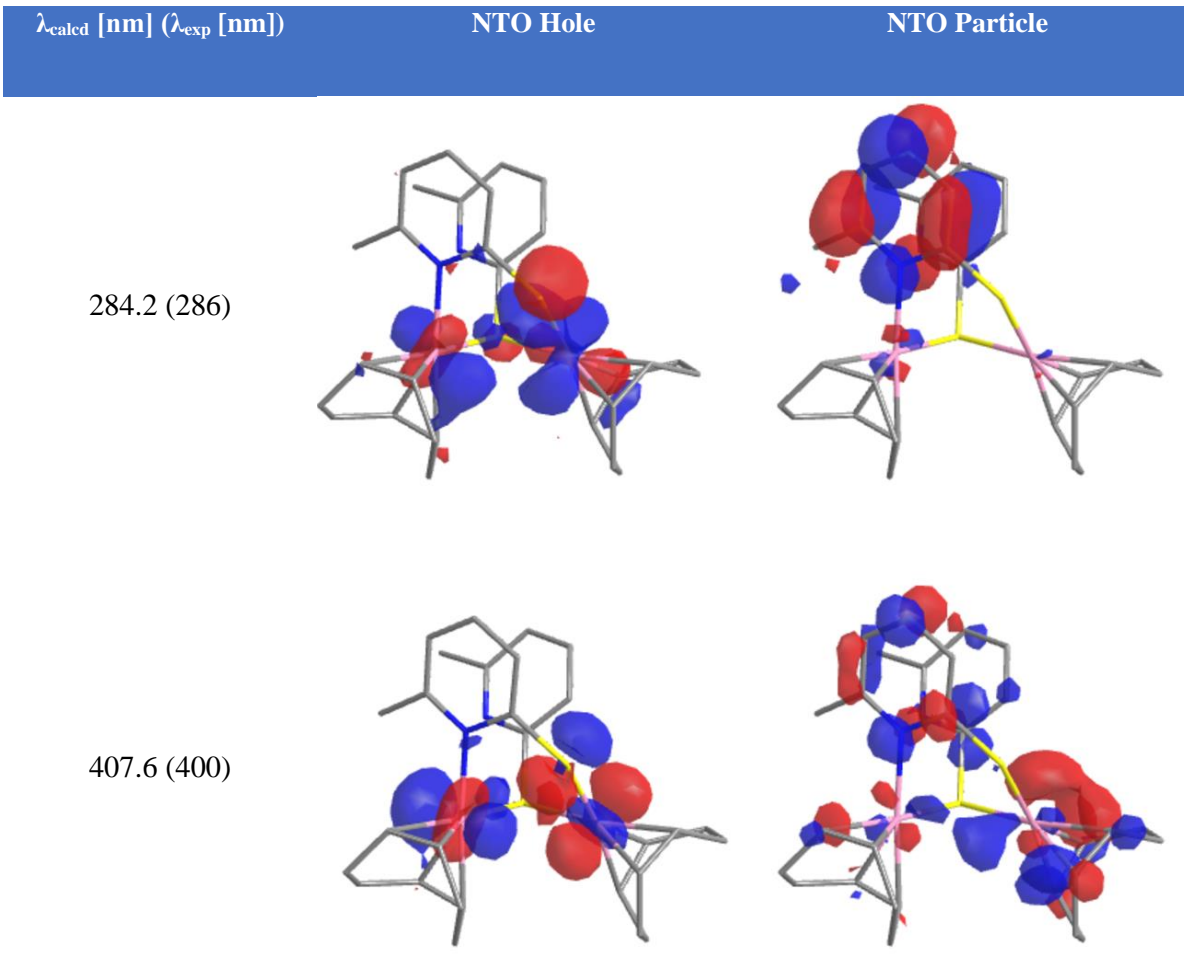




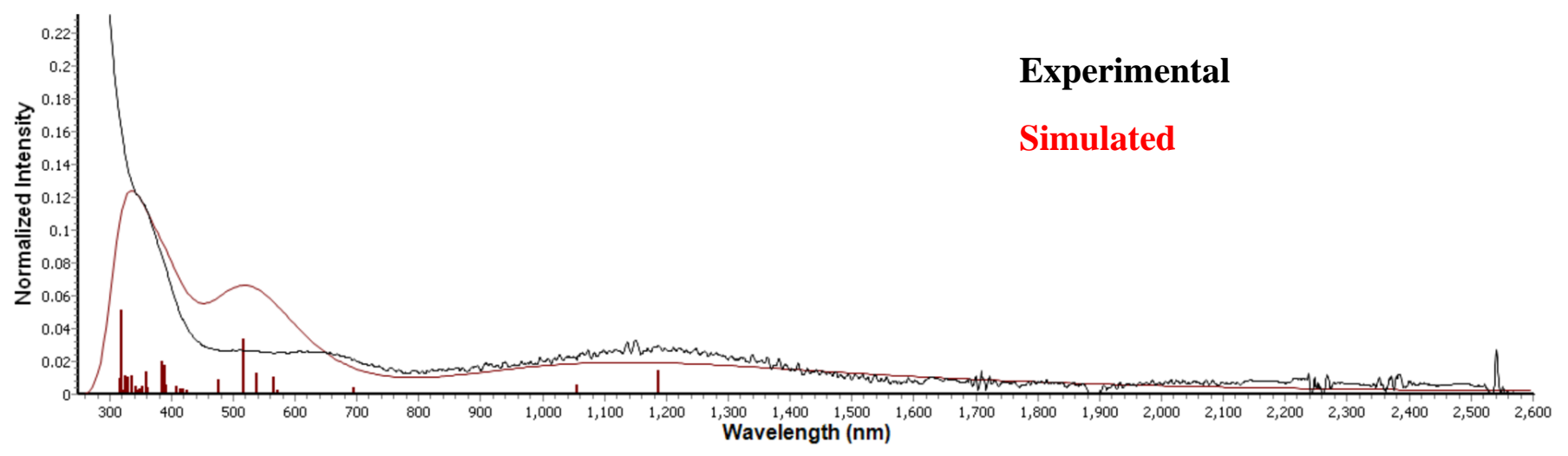

Figure S21. Experimental and TD-DFT calculated electronic absorption spectrum of 2.

Table S24. TD-DFT calculated excited states of 2.

\begin{tabular}{|c|c|c|c|}
\hline$\lambda_{\exp }(\mathrm{nm})$ & $\lambda_{\text {calcd }}(\mathbf{n m})$ & $f$ & Major Contributions \\
\hline 360 & 360.6 & 0.014 & $\begin{array}{c}\alpha \text { HOMO- } \rightarrow \rightarrow \text { LUMO }(33.6 \%) \\
\alpha \text { HOMO-7 } \rightarrow \text { LUMO }(33.6 \%) \\
\beta \text { HOMO- } 6 \rightarrow \text { LUMO }+1(32 \%)\end{array}$ \\
\hline 690 & 694.9 & 0.004 & $\begin{array}{c}\beta \text { HOMO- } 8 \rightarrow \text { LUMO }(125 \%) \\
\beta \text { HOMO- } 2 \rightarrow \text { LUMO }(22 \%)\end{array}$ \\
\hline \multirow[t]{2}{*}{1187} & 1056.7 & 0.006 & $\begin{array}{c}\beta \text { HOMO } \rightarrow \text { LUMO }(95 \%) \\
\beta \text { HOMO } 1 \rightarrow \text { LUMO }(92 \%)\end{array}$ \\
\hline & 1186.5 & 0.014 & $\begin{array}{l}\beta \text { HOMO } \rightarrow \text { LUMO }(104 \%) \\
\beta \text { HOMO- } 1 \rightarrow \text { LUMO }(82 \%)\end{array}$ \\
\hline
\end{tabular}

Table S25. NTO's of TD-DFT calculated excited states of $\mathbf{2}$ in the UV/vis region (isovalue $=0.04$ ).

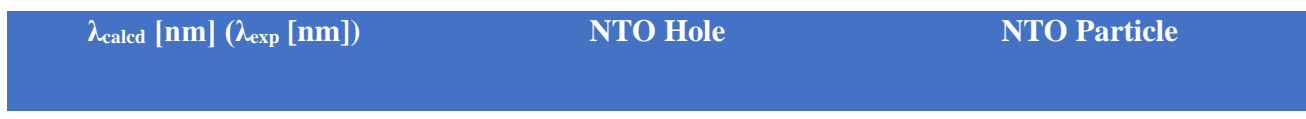

$360.6(360)$
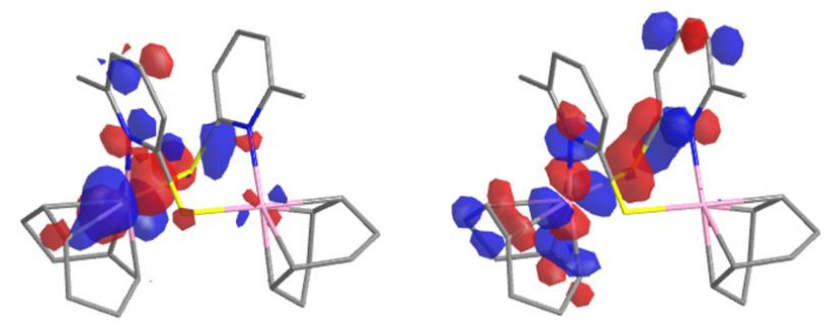

$694.9(690)$
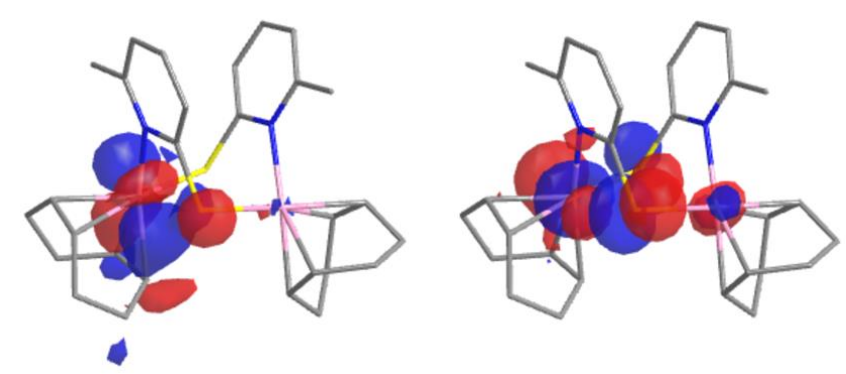


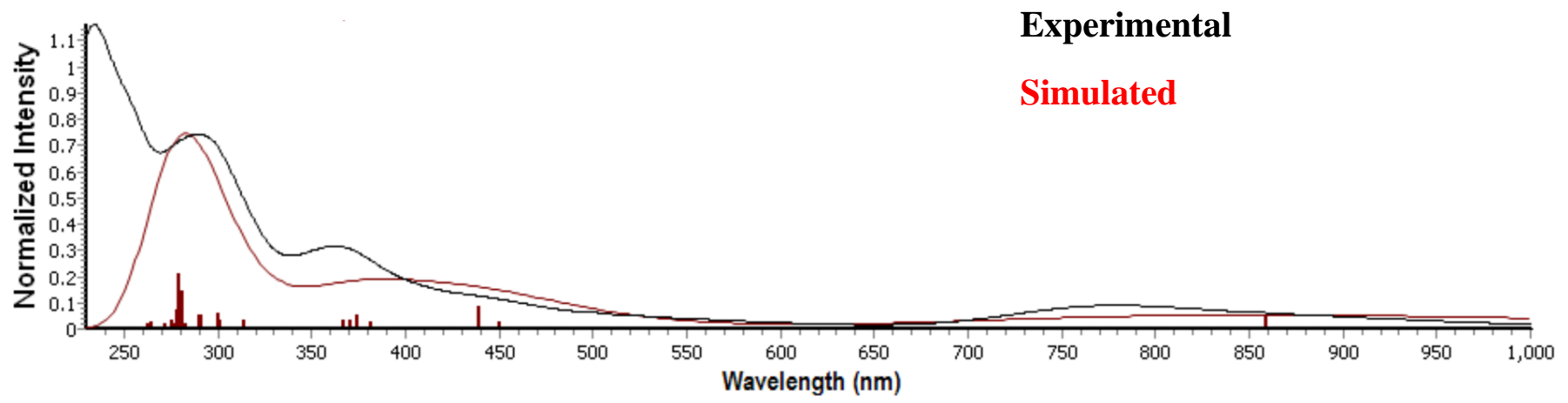

Figure S22. Experimental and TD-DFT calculated electronic absorption spectrum of 3.

Table S26. TD-DFT calculated excited states of $\mathbf{3}$.

\begin{tabular}{|c|c|c|c|}
\hline$\lambda_{\exp }(\mathrm{nm})$ & $\lambda_{\text {calcd }}(\mathrm{nm})$ & $f$ & Major Contributions \\
\hline 290 & 291.1 & 0.054 & $\begin{array}{c}\mathrm{HOMO} \rightarrow \mathrm{LUMO}+5(85 \%) \\
\mathrm{HOMO}-2 \rightarrow \mathrm{LUMO}+1(50 \%)\end{array}$ \\
\hline 362 & 366.9 & 0.036 & $\begin{array}{l}\text { HOMO- } \rightarrow \text { LUMO+2 }(74 \%) \\
\text { HOMO- } \rightarrow \text { LUMO+1 }(27 \%) \\
\text { HOMO-3 } \rightarrow \text { LUMO+1 }(23 \%) \\
\text { HOMO-3 } \rightarrow \text { LUMO+2 }(22 \%)\end{array}$ \\
\hline 779 & 859 & 0.053 & $\mathrm{HOMO} \rightarrow$ LUMO $(184 \%)$ \\
\hline
\end{tabular}

Table S27. NTO's of TD-DFT calculated excited states of $\mathbf{3}$ (isovalue=0.04).

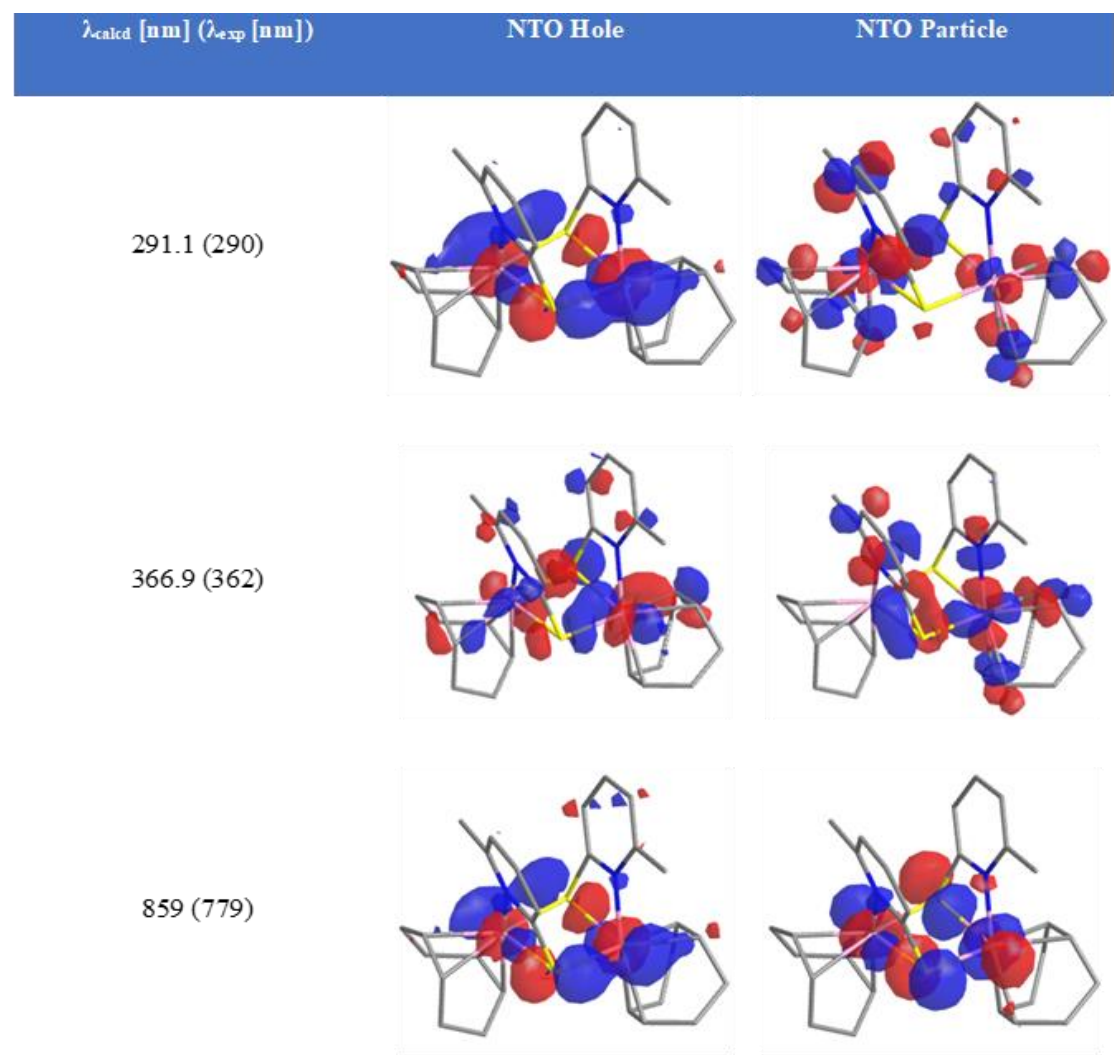




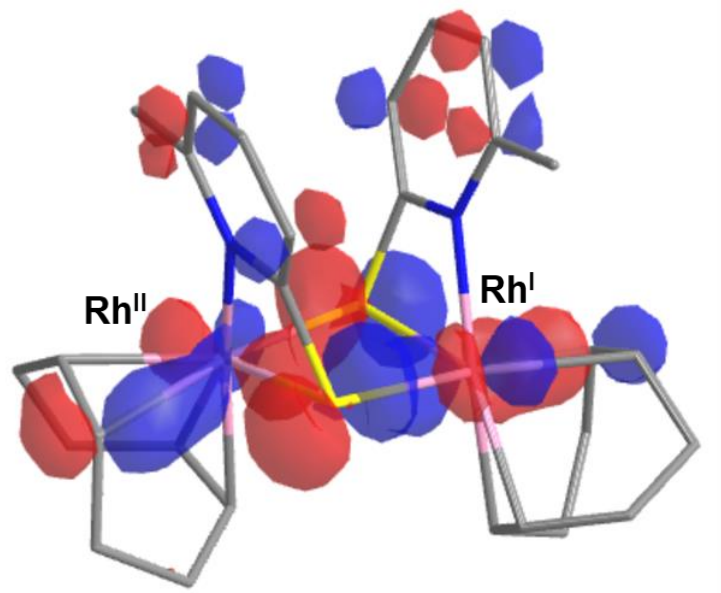

Hole

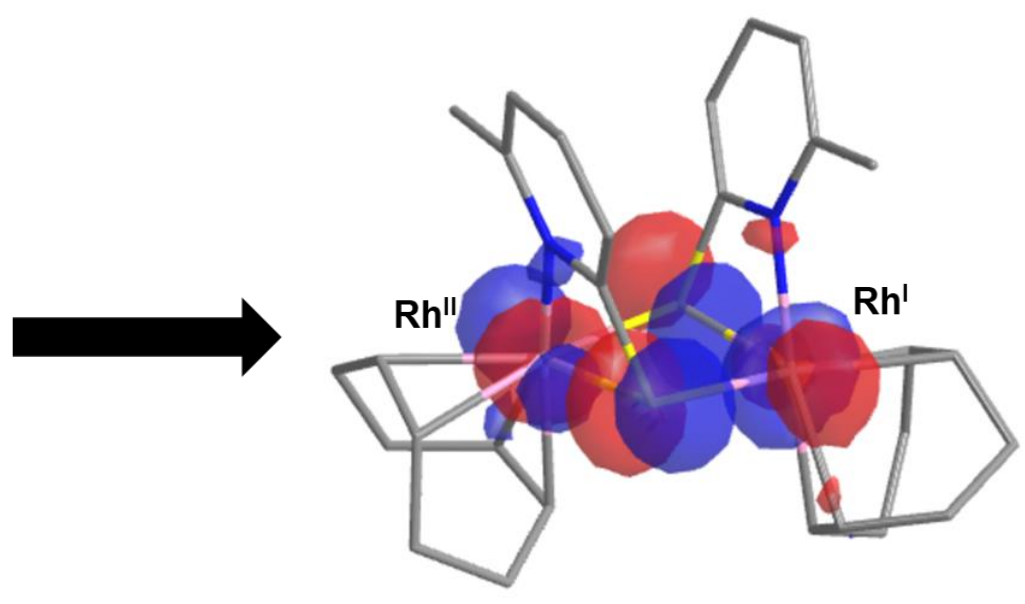

Particle

Figure S23. Calculated NTO's for a calculated triplet excited state transition in $\mathbf{3}$ at $\lambda_{\text {calc }}=1138.9 \mathrm{~nm}(f=0.00,92 \%$ contribution from HOMO- $\rightarrow$ LUMO transition, isovalue=0.04).

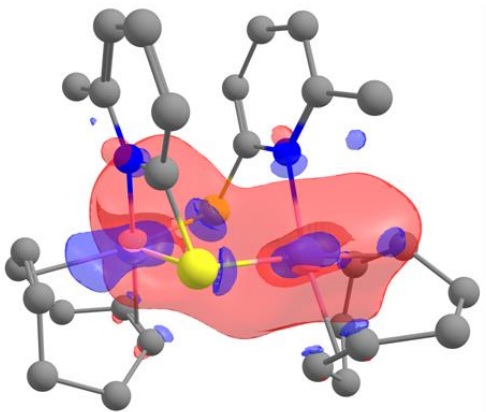

$20.36 \mathrm{kcal} / \mathrm{mol}$

Alpha-Rh2 $\left(5 p_{x / z}{ }^{0.405} s^{0.36} 4 d_{x 2-y 2 / y z}{ }^{0.24}\right)-\operatorname{Rh} 1\left(5 s^{0.95} 4 d_{x 2-y 2 / y z}{ }^{0.05}\right)$

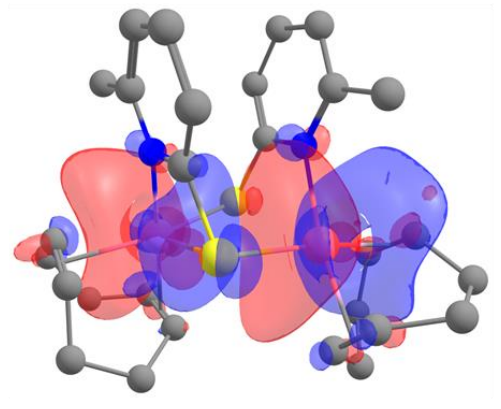

$18.39 \mathrm{kcal} / \mathrm{mol}$ Alpha-Rh2 $\left(5 p_{x / z}{ }^{0.955} 5 s^{0.04}\right)-\operatorname{Rh} 1\left(5 p_{x / z}{ }^{0.97} 4 d_{x z}{ }^{0.03}\right)$

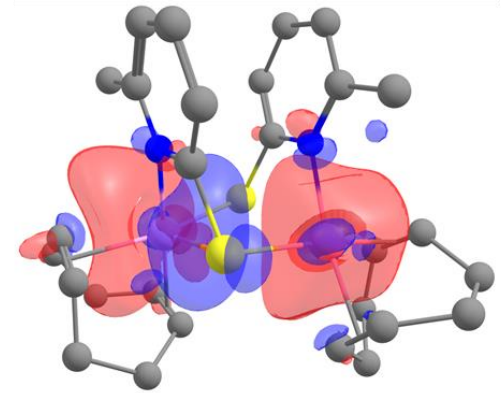

$10.48 \mathrm{kcal} / \mathrm{mol}$ Alpha-Rh1 $\left(5 s^{0.95} 4 d_{x 2-y 2 / y z}{ }^{0.05}\right)-R h 2\left(5 p_{x / z}{ }^{0.95} 5 s^{0.04}\right)$

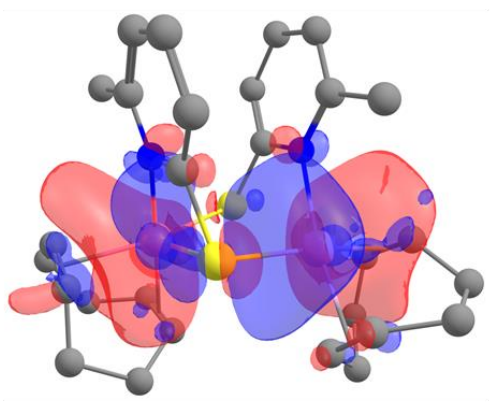

$49.68 \mathrm{kcal} / \mathrm{mol}$

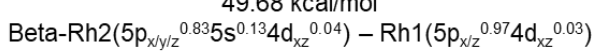

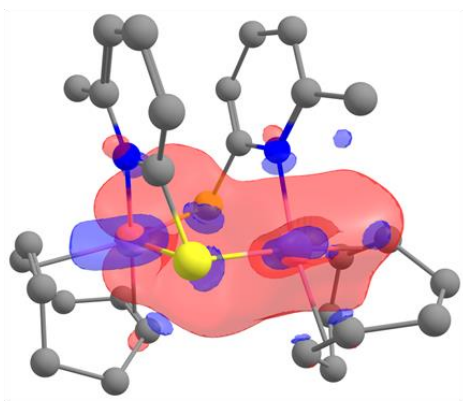

$27.68 \mathrm{kcal} / \mathrm{mol}$

Beta-Rh2 $\left(5 s^{0.37} 4 d_{x 2-y 2 / y z / z 2}{ }^{0.32} 5 p_{x}^{0.31}\right)-R h 1\left(5 s^{0.95} 4 d_{x 2-y 2 / y z}{ }^{0.05}\right)$

Figure S24. Natural bond order diagrams depicting Rh-Rh interactions in 2 (isovalue=0.04). 


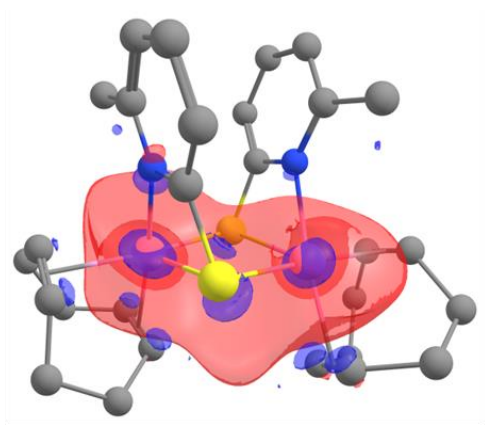

$95.42 \mathrm{kcal} / \mathrm{mol}$ Alpha-Rh1 $\left(5 s^{0.91} 4 d_{x 2-y 2 / y z}{ }^{0.06} 5 p_{x / z}{ }^{0.03}\right)-\operatorname{Rh} 2\left(5 s^{0.94} 4 d_{x 2-y 2}{ }^{0.035} 5 p_{z}^{0.02}\right)$

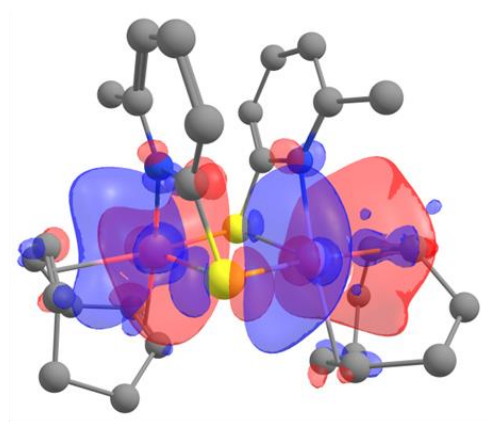

$79.56 \mathrm{kcal} / \mathrm{mol}$ Alpha-Rh2 $\left(5 p_{x / y / z}{ }^{0.99}\right)-\operatorname{Rh} 1\left(5 p_{x / z}{ }^{0.97} 4 d_{x 2-y 2}{ }^{0.02}\right)$

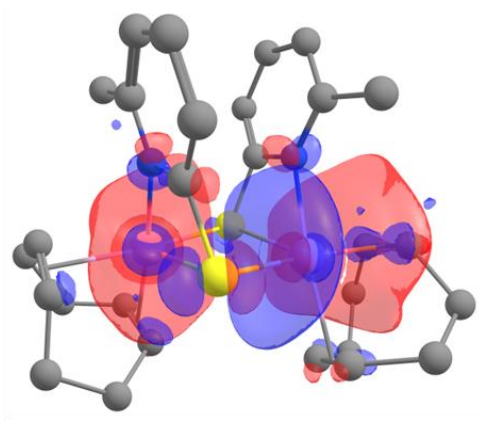
$\quad \begin{aligned} & 32.42 \mathrm{kcal} / \mathrm{mol} \\ & \text { Alpha-Rh2 }\left(5 \mathrm{~s}^{0.94} 4 \mathrm{~d}_{\mathrm{x} 2-\mathrm{y} 2} 2^{0.035} 5 \mathrm{p}_{\mathrm{z}}^{0.02}\right)-\operatorname{Rh} 1\left(5 \mathrm{p}_{\mathrm{x} / \mathrm{z}}{ }^{0.97} 4 \mathrm{~d}_{\mathrm{x} 2-\mathrm{y} 2}{ }^{0.02}\right)\end{aligned}$

Figure S25. Natural bond order diagrams depicting Rh-Rh interactions in $\mathbf{3}$ (isovalue $=0.04$ ).

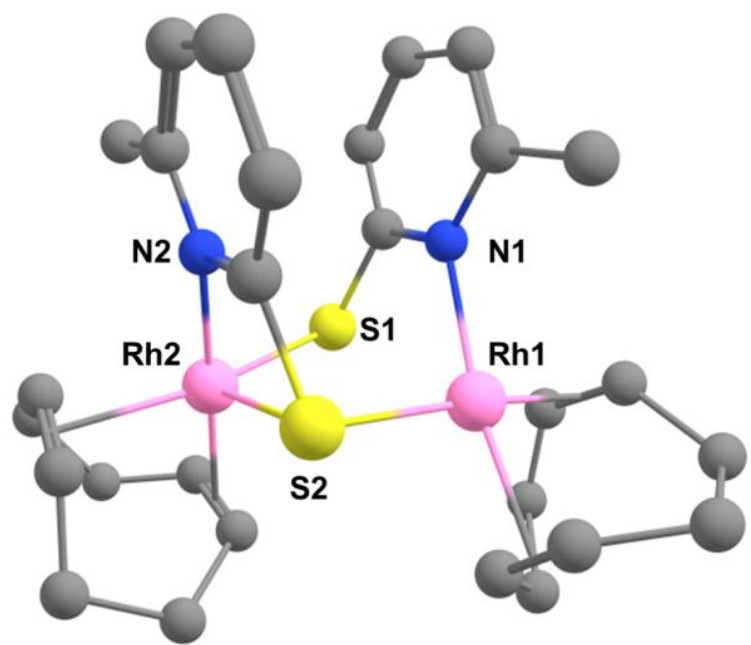

Table S28. Calculated spin density values for Rh, S, and $\mathrm{N}$ atoms of 2.

$\begin{array}{cc}\text { Atom } & \text { Spin Density } \\ \text { Rh1 } & 0.036384 \\ \text { Rh2 } & 0.537447 \\ \text { S1 } & 0.158960 \\ \text { S2 } & 0.256335 \\ \text { N1 } & 0.001364 \\ \text { N2 } & 0.023953\end{array}$




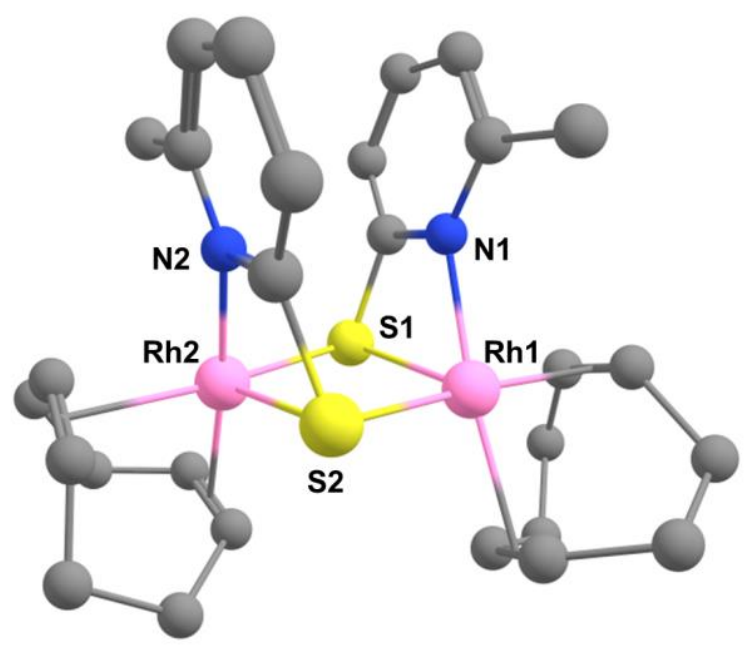

Table S29. Calculated spin density values for Rh, S, and N atoms of 3.

$\begin{array}{cc}\text { Atom } & \text { Spin Density } \\ \text { Rh1 } & 0.659778 \\ \text { Rh2 } & 0.654865 \\ \text { S1 } & 0.298003 \\ \text { S2 } & 0.299277 \\ \text { N1 } & 0.031687 \\ \text { N2 } & 0.030136\end{array}$

Table S30. Computed $J$ values for complex $\mathbf{3}$ using various functionals along with the spin density on each Rh center resulting from broken symmetry (BS) and high spin (HS) calculations.

\begin{tabular}{cccccc} 
Functional & $J\left(\mathrm{~cm}^{-1}\right)$ & \multicolumn{2}{c}{ Rh Spin Density (BS) } & \multicolumn{2}{c}{ Rh Spin Density (HS) } \\
uB3LYP & -1516.9 & -0.516615 & 0.512527 & 0.659777 & 0.654865 \\
uM06 & -1885.2 & -0.333843 & 0.327913 & 0.642826 & 0.637352 \\
uTPSSh & -1664.1 & -0.466539 & 0.461801 & 0.670099 & 0.665077 \\
uB3LYP-D3 & -1516.9 & -0.516615 & 0.512528 & 0.659776 & 0.654865
\end{tabular}




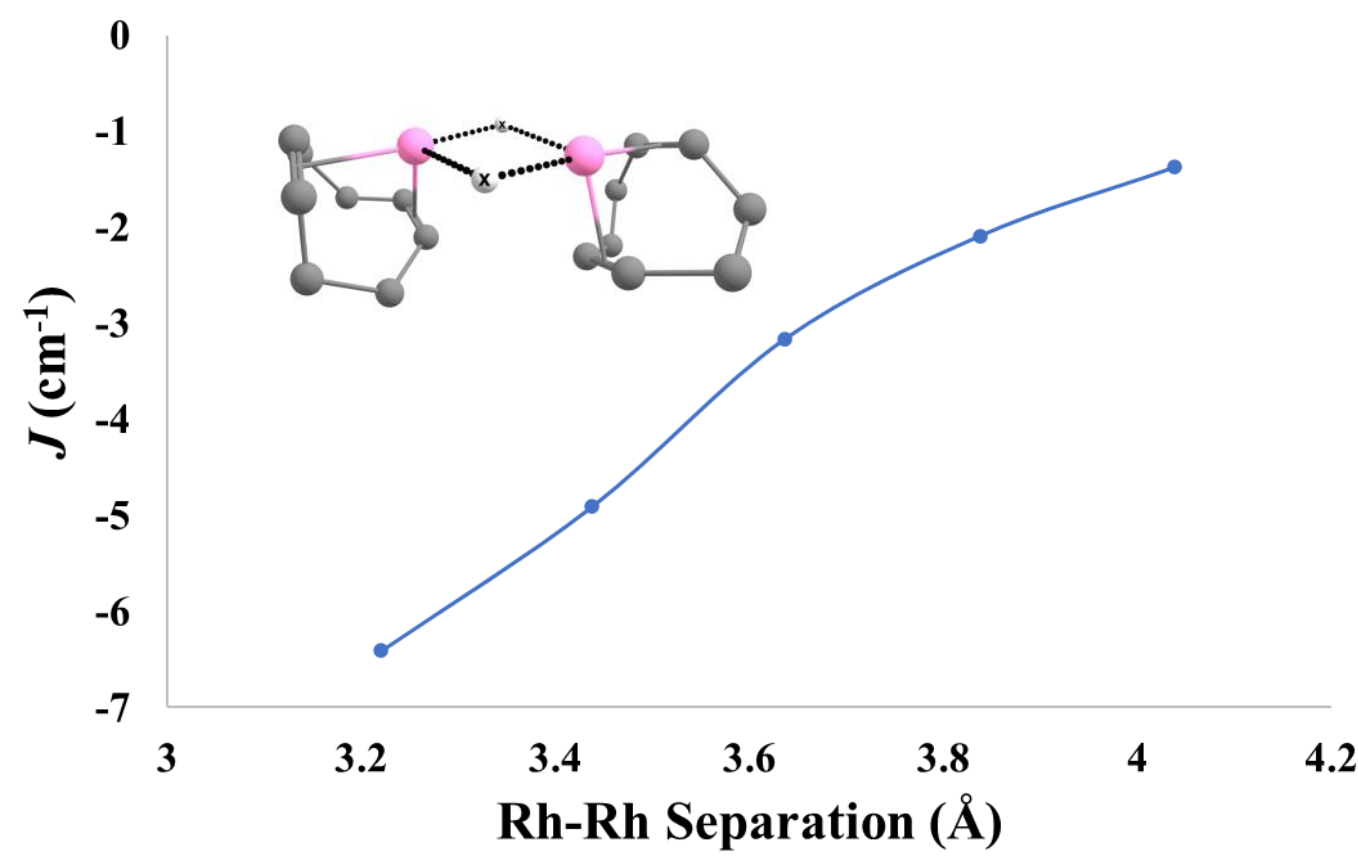

Figure S26. Magneto-structural correlation between Rh-Rh distance in $\mathbf{3}$ and the resulting calculated $J$ value when the bridging pyridyl groups are replaced with a negative point charge at the cartesian coordinate site of the $\mathrm{S}$ atoms (Inset: 3 crystal structure with pyridyl groups replaced by " $\mathrm{X}$ " which denotes the Mulliken point charge).

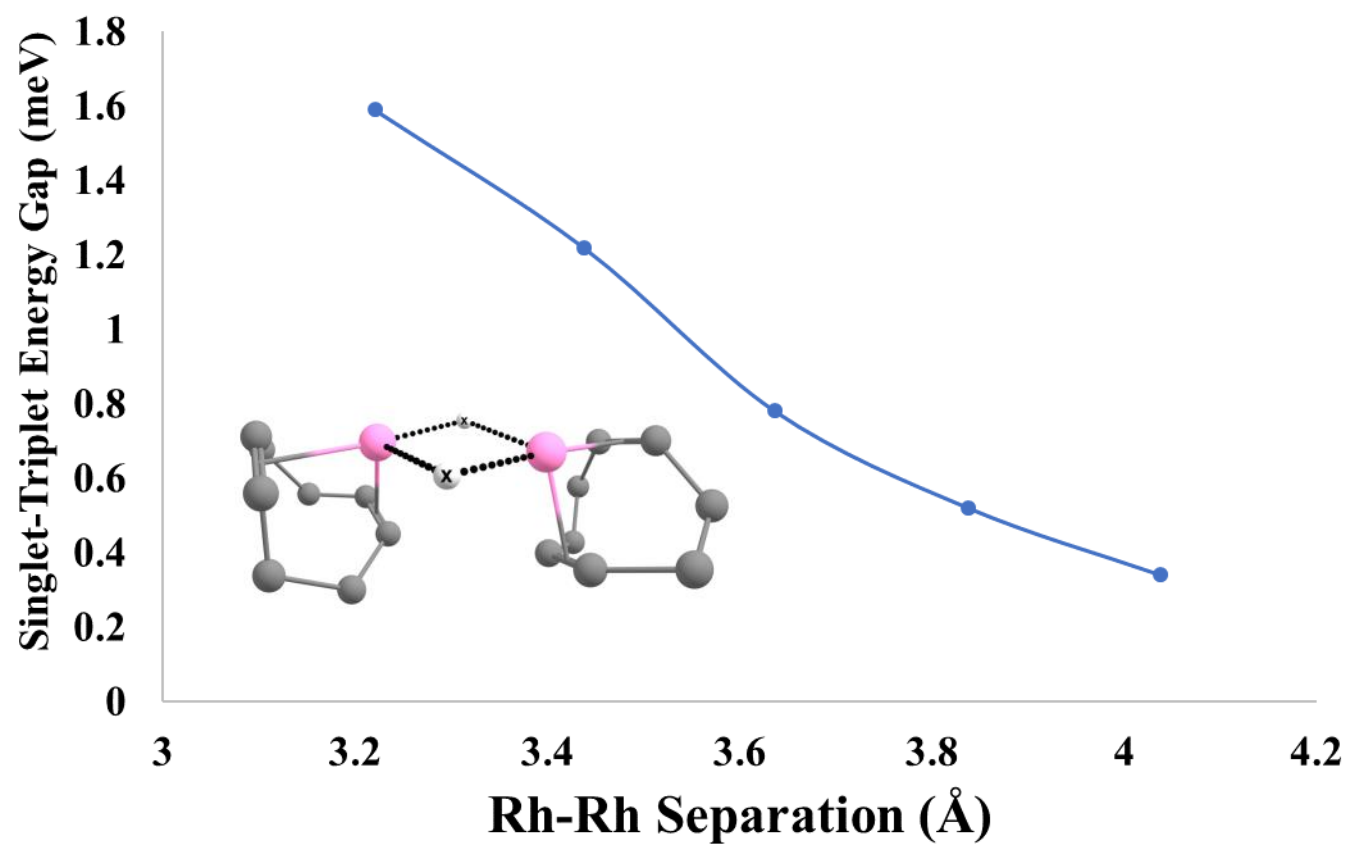

Figure S27. Magneto-structural correlation between $\mathrm{Rh}-\mathrm{Rh}$ distance in $\mathbf{3}$ and the resulting singlet-triplet energy gap when the bridging pyridyl groups are replaced with a negative point charge at the cartesian coordinate site of the $\mathrm{S}$ atoms (Inset: 3 crystal structure with pyridyl groups replaced by "X" which denotes the Mulliken point charge). 